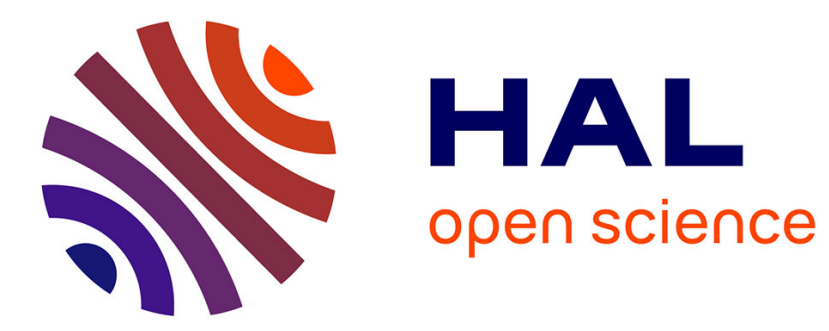

\title{
Plant seeds as model vectors for the transfer of life through space
}

\author{
David Tepfer, Sydney Leach
}

\section{To cite this version:}

David Tepfer, Sydney Leach. Plant seeds as model vectors for the transfer of life through space. Astrophysics and Space Science, 2006, 306 (1-2), pp.69-75. 10.1007/s10509-006-9239-0 . hal-02659643

\section{HAL Id: hal-02659643 \\ https://hal.inrae.fr/hal-02659643}

Submitted on 30 May 2020

HAL is a multi-disciplinary open access archive for the deposit and dissemination of scientific research documents, whether they are published or not. The documents may come from teaching and research institutions in France or abroad, or from public or private research centers.
L'archive ouverte pluridisciplinaire HAL, est destinée au dépôt et à la diffusion de documents scientifiques de niveau recherche, publiés ou non, émanant des établissements d'enseignement et de recherche français ou étrangers, des laboratoires publics ou privés. 


\title{
Hypothesis Paper
}

\section{Experimentally Tracing the Key Steps in the Origin of Life: The Aromatic World}

\author{
PASCALE EHRENFREUND, ${ }^{1}$ STEEN RASMUSSEN, ${ }^{2,3,4}$ JAMES CLEAVES, $^{5}$ \\ and LIAOHAI CHEN ${ }^{6}$
}

\begin{abstract}
Life is generally believed to emerge on Earth, to be at least functionally similar to life as we know it today, and to be much simpler than modern life. Although minimal life is notoriously difficult to define, a molecular system can be considered alive if it turns resources into building blocks, replicates, and evolves. Primitive life may have consisted of a compartmentalized genetic system coupled with an energy-harvesting mechanism. How prebiotic building blocks self-assemble and transform themselves into a minimal living system can be broken into two questions: (1) How can prebiotic building blocks form containers, metabolic networks, and informational polymers? (2) How can these three components cooperatively organize to form a protocell that satisfies the minimal requirements for a living system? The functional integration of these components is a difficult puzzle that requires cooperation among all the aspects of protocell assembly: starting material, reaction mechanisms, thermodynamics, and the integration of the inheritance, metabolism, and container functionalities. Protocells may have been self-assembled from components different from those used in modern biochemistry. We propose that assemblies based on aromatic hydrocarbons may have been the most abundant flexible and stable organic materials on the primitive Earth and discuss their possible integration into a minimal life form. In this paper we attempt to combine current knowledge of the composition of prebiotic organic material of extraterrestrial and terrestrial origin, and put these in the context of possible prebiotic scenarios. We also describe laboratory experiments that might help clarify the transition from nonliving to living matter using aromatic material. This paper presents an interdisciplinary approach to interface state of the art knowledge in astrochemistry, prebiotic chemistry, and artificial life research. Key Words: Origin of lifeAromatic hydrocarbons-Minimal life-Young Earth. Astrobiology 6, 490-520.
\end{abstract}

\footnotetext{
${ }^{1}$ Leiden Institute of Chemistry, Leiden, The Netherlands.

${ }^{2}$ Los Alamos National Laboratory, Los Alamos; and ${ }^{3}$ Santa Fe Institute, Santa Fe, New Mexico.

${ }^{4}$ University of Copenhagen, IMBG Panum Institute, Kobenhavn, Denmark.

${ }^{5}$ Geosciences Research Division, Scripps Institution of Oceanography, La Jolla, California.

${ }^{6}$ Bioscience Division, Argonne National Laboratory, Argonne, Illinois.
} 


\section{INTRODUCTION}

$\mathbf{I}^{\mathrm{T}}$ IS GENERALLY AGREED that life began relatively rapidly after conditions on the surface of the Earth allowed it, at least 3.85 billion years ago (Mojzsis et al., 1996), though the evidence from the geological record is still under debate (Nisbet and Sleep, 2001; van Zuilen et al., 2002). Because very few data are available regarding the atmospheric, oceanic, or geological conditions on the pre-biological Earth, it is difficult to determine conclusively whether terrestrially synthesized organic material or extraterrestrial molecules were more significant for life's origin. A fundamental question remains as to how simple molecules of terrestrial or extraterrestrial origin assembled into larger functional units under plausible geochemical conditions and then into replicating structures. Advances in directed molecular evolution, molecular self-assembly, and artificial redox and photochemical metabolic systems make the synthesis of protocells an imaginable goal (Szostak et al., 2001; Rasmussen et al., 2004).

There are two fundamental approaches to the study of the origin of life. One, the top-down approach, considers the origin of the common components of modern biochemistry and their organization. The other, the bottom-up approach, considers which compounds may have been plausibly produced under primitive planetary conditions and how they came to self-assemble. The top-down approach is biased by the uniformity of modern biochemistry across the three extant domains of life (Archaea, Bacteria, and Eukarya), which clearly originated from a common ancestor (Pace, 2001; Zhaxybayeva and Gogarten, 2004). Whatever the first living being was, the origin of life likely depended on the presence of organic compounds. Understanding the source and nature of this material is crucial for our understanding of subsequent molecular organization.

The endogenous synthesis of prebiotic organic compounds depends on the conditions on the young Earth (e.g., atmospheric composition, oxidation state of the early mantle, hydrothermal systems, glaciation, etc.), which are unfortunately poorly understood. Recent evidence from zircon crystals suggests that surface temperatures were relatively low and liquid oceans were present rather than a thick steam-rich atmosphere (Wilde et al., 2001). Such oceans could have facilitated the evolution of life. The amount of organic material produced by atmospheric synthesis or hydrother- mal vent chemistry would have depended on the nature of the gases emitted by early volcanism. Extraterrestrial material is estimated to have delivered $\sim 10^{9} \mathrm{~kg}$ of carbon per year to the Earth during the heavy bombardment phase $4.5-3.8$ billion years ago (Chyba and Sagan, 1992). For comparison, endogenous synthesis is estimated to have produced between $1 / 100^{\text {th }}$ this value for neutral atmospheres to 1,000 times this value for highly reducing atmospheres (Stribling and Miller, 1987). Ehrenfreund et al. (2002) provided a recent quantitative estimate of major sources of organic compounds on the early Earth. If the early atmosphere was not reducing, then infalling material from space may have been the predominant source. As our knowledge of the composition of interstellar dust and gas, comets, asteroids, and meteorites grows we can determine more accurately the molecular inventory of material that was transported to young planets by exogenous delivery. Recent astronomical observations and laboratory analysis of extraterrestrial material suggest that the majority of carbon in the universe is in the form of aromatic solid macromolecular material (Ehrenfreund et al., 2004). Interstellar carbon chemistry proceeds similarly throughout the universe as evidenced by astronomical observations of galactic and extragalactic regions (e.g., Spoon et al., 2003; Peeters et al., 2005; Sollerman et al., 2005). Therefore, extraplanetary input of organic matter may proceed in similar ways in other solar systems as well.

Life must have initially been fairly simple and may have used the most abundant and stable material present on the young Earth. Astronomical observations of comets and laboratory measurements of carbonaceous meteorites show that the most abundant carbon material they likely carried to Earth were aromatic molecules. Aromatic molecules compacted in macromolecular carbon or as free volatiles may have formed on the early Earth as well (Gold, 1999). Aromatic molecules are typically very stable. Highly aromatic macromolecular carbon (such as that found in meteorites) may fragment and release soluble organics upon chemical oxidation at slightly elevated temperatures. Components active in modern biochemistry are fragile with respect to heat, radiation, and oxidation, and therefore the self-assembly of life's precursor molecules from a pool of solid and volatile aromatic structures may be a more realistic scenario under hostile early Earth conditions. 
In this paper we discuss the formation, inventory, and development of cosmic starting material for the origin of life in interstellar space (The Formation and Evolution of Organic Material in Space) and in the solar system (From the Interstellar Medium to the Terrestrial Planets). Terrestrial sources of organic matter and possible prebiotic scenarios on the young Earth are summarized in Terrestrial Sources of Organic Matter and Possible Prebiotic Scenarios. Despite many decades of laboratory efforts in prebiotic chemistry (using components present in contemporary life) no definitive origin of biochemistry scenario has been elaborated. In Functional Assemblies and Their Integration into a Minimal Life Form, we propose how assemblies of aromatic hydrocarbons might be integrated into a minimal life form. We discuss laboratory experiments that could provide insights into this hypothesis and suggest that the first terrestrial life may have been composed of compounds no longer used in modern biochemistry.

\section{THE FORMATION AND EVOLUTION OF ORGANIC MATERIAL IN SPACE}

To understand how life originated on Earth, it is useful to review several crucial steps in the evolution of the universe such as the formation of the chemical elements, molecules and cosmic dust, and the processes that led to the formation of planets. Observations of numerous protoplanetary disks and the detection of more than 170 extrasolar planets provide evidence that planets are common in the universe (see http://exoplanets.org/) (Marcy et al., 2005). The detection of small terrestrial planets will require further technological advances, though recently a frozen Earth-like planet, OGLE-2005BLG-390Lb, has been discovered during a gravitational microlensing campaign (Beaulieu et al., 2006). Earth has provided unique conditions for life such as moderate surface temperatures, abundant water, a stable orbit and inclination, and a dynamic atmosphere and interior structure-properties that may be uncommon on other planets (Ehrenfreund and Martin, 2006). Organic chemistry in space, however, seems to follow common pathways throughout the universe. Carbonaceous molecules in the gas or solid state (carbonaceous dust or icy grains) are observed in similar abundances and composition in our and distant galaxies (Spoon et al., 2003; Peeters et al., 2005; Sollerman et al., 2005)
(Fig. 1). In the last decade astronomical observations have allowed us to peer into distances of many billions of light years. Such observations of galaxies at high redshift probe the early universe and show that carbonaceous molecules were already present and dust formation already occurring (Bertoldi et al., 2003; Kaneda et al., 2005; Yan et al., 2005). Taking into account the dimensions of our universe, the ubiquitous observations of aliphatic and aromatic molecules, as well as C-bearing ices (such as $\mathrm{CO}$ ), provide a clear indication that chemical processes have proceeded in similar ways. Such factors also compel us to consider Life in the larger context of astrophysics. The largest fraction of carbon in the universe is incorporated into solid aromatic macromolecular matter. Few studies have been performed with regard to how such material could have been involved in the origin of life. Insights into organic chemistry in space will assist our ability to predict whether life as we know it is likely to exist on other planets as well.

\section{Formation of the elements}

The universe is estimated to be approximately 13.7 billion years old. During Big Bang nucleosynthesis, only $\mathrm{H}$ and $\mathrm{He}$ and traces of a few other light nuclei such as $\mathrm{D}, \mathrm{T}, \mathrm{Li}$, and Be were formed. Since the universe was expanding, cooling, and decreasing in density, heavier elements could not have formed during its early history. Carbon, the basis of organic chemistry, is produced by the so-called triple- $\alpha$ process (3 ${ }^{4} \mathrm{He} \rightarrow{ }^{12} \mathrm{C}$ ) in the cores of stars. Today, stars more massive than half a solar mass form carbon after they have fused a significant fraction of their core hydrogen into helium. In the same cores, some carbon is further processed to the principal isotope of oxygen by ${ }^{12} \mathrm{C}+{ }^{4} \mathrm{He} \rightarrow{ }^{16} \mathrm{O}$. During the stellar burning cycle, contraction and heating of the stellar core alternate with a cycle of expansion and cooling. In this way, heavier elements (until ${ }^{56} \mathrm{Fe}$ ) are produced in more massive stars. ${ }^{56} \mathrm{Fe}$ has the greatest nuclear binding energy and is unable to fuse with other nuclei to produce energy. The accumulation of ${ }^{56} \mathrm{Fe}$ in the stellar core destabilizes the star and triggers stellar collapse. All elements heavier than iron are formed during the final evolutionary period of stars dominated by mass loss, core degradation, and stellar explosions. The cosmic abundances of the chemical elements are summarized in Table 1. New perspectives on cosmic abun- 
FIG. 1. More than 300 diffuse interstellar bands (DIBs) are observed in the absorption spectra of stars distributed throughout the universe. Those bands most probably originate from large carbonaceous molecules such as PAHs or fullerenes (Salama et al., 1996). Two strong DIBs appear at 5780 and $5797 \AA$ in the visible range. The signature of these bands can be observed in galactic and extragalactic regions. Here we display an example of those bands in our Milky Way, our neighbor galaxies the Large and Small Magellanic Clouds, and toward a supernova in a distant galaxy, NGC 1448.

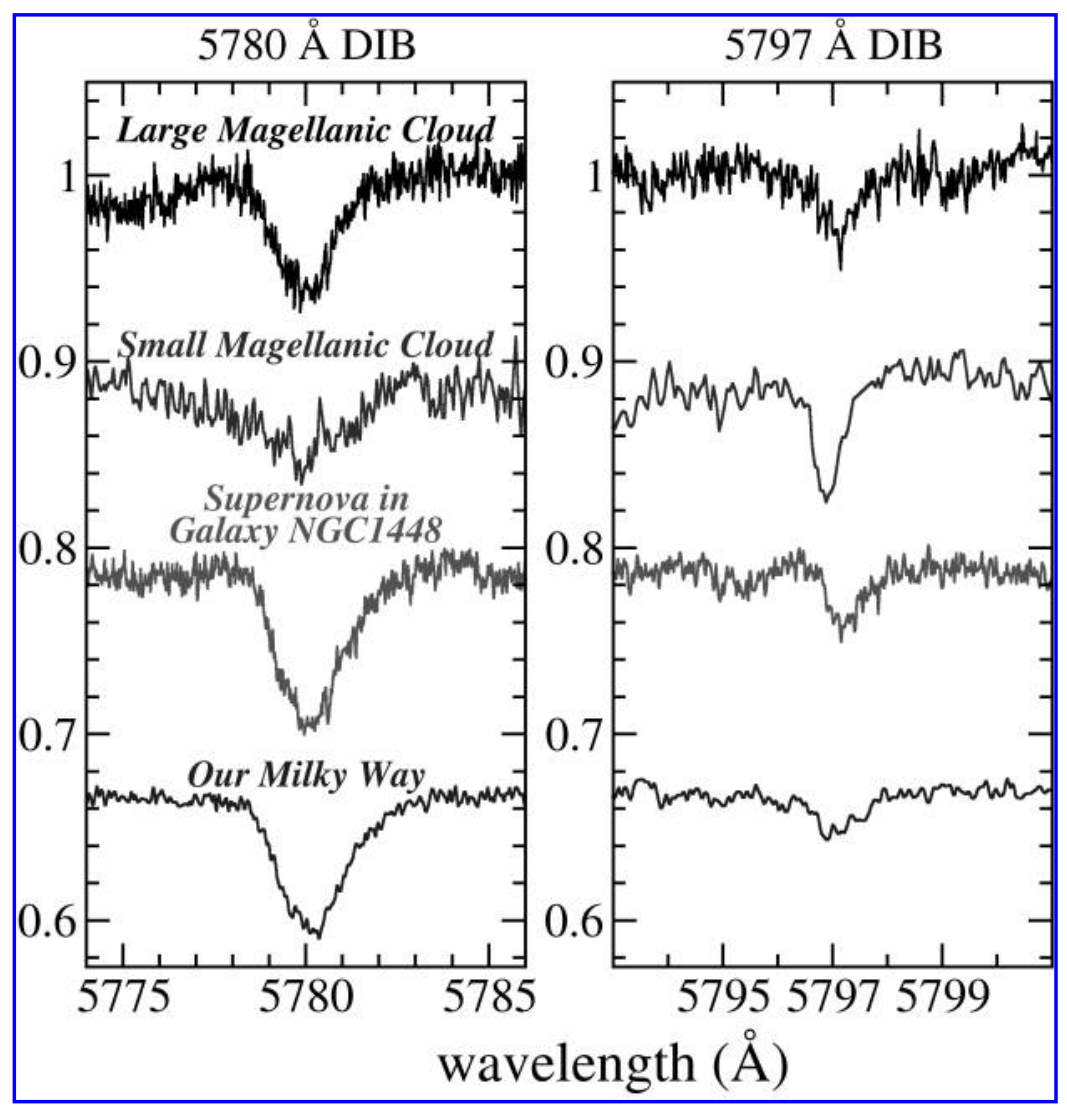

dances have been recently summarized by Beers (2005).

\section{The first stars}

Recent cosmological data indicate that the formation of the first stars (population III non-metallic stars) may have occurred as early as 200 million years after the Big Bang (Daigne et al., 2004). These very massive stars (probably more than 100

Table 1. Elemental Abundances in Space Relative to H

\begin{tabular}{lc}
\hline Element & Abundance \\
\hline $\mathrm{H}$ & 1 \\
$\mathrm{He}$ & 0.1 \\
$\mathrm{O}$ & $8.3 \times 10^{-4}$ \\
$\mathrm{C}$ & $4.0 \times 10^{-4}$ \\
$\mathrm{~N}$ & $1.0 \times 10^{-4}$ \\
$\mathrm{~S}$ & $1.7 \times 10^{-5}$ \\
$\mathrm{P}$ & $3.0 \times 10^{-7}$ \\
$\mathrm{Ne}$ & $0.8 \times 10^{-4}$ \\
$\mathrm{Si}$ & $4.3 \times 10^{-5}$ \\
$\mathrm{Mg}$ & $4.2 \times 10^{-5}$ \\
$\mathrm{Fe}$ & $4.3 \times 10^{-5}$ \\
$\mathrm{Na}$ & $2.1 \times 10^{-6}$ \\
\hline
\end{tabular}

solar masses) were born from gravitationally contracting gas clouds dominated by hydrogen and helium in dark-matter halos (Scannapieco et al., 2003). These stars can no longer be observed. The recent detection of hyper-metal-poor (HMPs) stars provides important constraints for early star formation processes (Iwamoto et al., 2005). These HMP stars have a $\mathrm{Fe} / \mathrm{H}$ ratio less than $[1 / 100,000]$ of the solar ratio. They have an overabundance in $\mathrm{C}$ and $\mathrm{O}$ relative to $\mathrm{Fe}$, and are slightly less massive than the Sun (Iwamoto et al., 2005; Spaans and Silk, 2005). Investigations of those stars may provide us with clues with regard to the first generation of stars and supernovae that distributed the first heavy elements (Umeda and Nomoto, 2005) in the early universe.

The cycle of birth and death of stars constantly increases the abundance of heavy elements in the interstellar medium, a crucial prerequisite for terrestrial (rocky) planet formation and subsequently for the origin of life (for a review, see Spaans, 2004). In astronomical language, elements heavier than $\mathrm{H}$ and $\mathrm{He}$ are called "metals." Metals dispersed in the interstellar gas or incorporated into micron-sized dust particles have the ability to efficiently cool the interstellar gas. 
Those elements are excited by molecular collisions, and their return to stable energy levels releases energy via ultraviolet (UV) radiation in a much more efficient way than $\mathrm{H}$ collisions. In turn, cooling of the interstellar gas influences cloud fragmentation and triggers the formation of low-mass stars like our Sun (Scalo and Biswas, 2002). Low-mass stars have long life spans (e.g., the Sun has a stable lifetime of $\sim 10$ billion years) that allow sufficient time to form terrestrial planets and life to evolve.

Understanding the early universe is extremely relevant to the question of terrestrial planet formation and consequently life. Observations and theory in the last decade indicate that star formation and heavy element enrichment occurred much earlier than previously thought (Daigne et al., 2004).

\section{From elements to molecules}

Interstellar clouds and circumstellar envelopes act as nurseries for complex molecules and for siliceous and carbonaceous micron-sized dust particles. The interstellar medium constitutes $\sim 10 \%$ of the mass of the galaxy. The interstellar medium can be subdivided into environments with very low-density hot gas, environments with warm intercloud gas, and regions with denser and colder material (Wooden et al., 2004). $\mathrm{H}$ and $\mathrm{He}$ gas are the major components of interstellar clouds; molecules and submicron dust particles are only present in low concentration (Ehrenfreund and Charnley, 2000). Complex organic molecules can be formed through gasphase reactions, solid-state chemistry, and gasgrain interactions. $\mathrm{H}_{2}$ is by far the most abundant molecule in these clouds. $\mathrm{CO}$ is the most abundant carbon-containing species, with $\mathrm{CO} / \mathrm{H}_{2} \sim$ $10^{-4}$. High excitation CO emissions from the most distant quasar currently known at redshift $z=$ 6.419 have been detected, which indicates that $\mathrm{CO}$ has been present in the universe since approximately 800 millions years after the Big Bang (Bertoldi et al., 2003). Silicate and carbon-based micron-sized dust particles, which are produced in the outflows of late-type stars, provide a catalytic surface for a variety of reactions when they are dispersed in molecular clouds (Ehrenfreund and Fraser, 2003). In cold clouds such dust particles adsorb ice mantles of water, $\mathrm{CO}_{2}, \mathrm{CO}$, and $\mathrm{CH}_{3} \mathrm{OH}$, with smaller admixtures of $\mathrm{CH}_{4}, \mathrm{NH}_{3}$, $\mathrm{H}_{2} \mathrm{CO}$, and $\mathrm{HCOOH}$. Observations at infrared, radio, millimeter, and submillimeter frequencies show that a large variety of organic molecules are present in the dense interstellar gas (Charnley et al., 2003). These include organics such as nitriles, aldehydes, alcohols, acids, ethers, ketones, amines, and amides, as well as long-chain hydrocarbons. The effective shielding of UV radiation by dust in such high-density regions enables complex gas-grain chemistry in the so-called "hot-core" regions close to the forming star, which allows the formation of many organic molecules, including precursor molecules for life as we know it (Kuan et al., 2003) (see http://www. astrochemistry.net).

In addition to the rich inventory of prebiotic species observed in the gas phase in dense starforming regions, laboratory experiments show the formation of amino acids upon UV irradiation of interstellar icy grain analogs (Bernstein et al., 2002; Muñoz Caro et al., 2002). Star-forming regions where interstellar icy grains evolve are strongly devoid of UV irradiation (Prasad and Tarafdar, 1983). Furthermore, it has been shown that prebiotic molecules such as amino acids and small heterocycles are rapidly degraded by UV radiation (Peeters et al., 2003, 2005). The onset of the star-formation process is violent and destructive to any volatile or fragile (even when embedded in solid) material. There is, thus, no likely direct connection between simple interstellar prebiotic material (such as amino acids, sugars, etc.) in dense clouds and the origin of life on Earth. Observations of comets have shown that all of the original interstellar material has been strongly processed in the solar nebula (Ehrenfreund et al., 2004). Only robust refractory material (such as silicates, metals, and solid carbonaceous matter) may retain significant interstellar heritage, as evidenced by isotopic enrichments (e.g., Clemett et al., 1993; Botta and Bada, 2002; Sephton et al., 2004).

Carbon chemistry occurs most efficiently in circumstellar and diffuse interstellar clouds. The circumstellar envelopes of carbon-rich stars are the heart of the most complex carbon chemistry, which is analogous to soot formation in candle flames or industrial smoke stacks (Henning and Mutschke, 2004). It is expected that carbon grains forming in the outflows of carbon-rich Asymptotic Giant Branch stars are composed of a wide variety of structures (Henning and Salama, 1998). Those stars are in a late stage of their evolution and lose mass via a dense and dusty outflow. 
Molecular synthesis occurs in the circumstellar environment on timescales as short as several hundred years (Kwok, 2004). Acetylene $\left(\mathrm{C}_{2} \mathrm{H}_{2}\right)$ appears to be the precursor for the synthesis of hexagonal aromatic rings of carbon atoms. Benzene detection has been claimed in the Infrared Space Observatory spectrum of the circumstellar envelope CRL 618 (Cernicharo et al., 2001). Recent laboratory data have shown that benzene could be available for aromatic chemistry when sufficiently shielded in circumstellar envelopes from protons and UV photons (Ruiterkamp et al., 2005a). Benzene chemistry is the first step in the formation of polycyclic aromatic hydrocarbons (PAHs), fullerene-type material, and larger aromatic macromolecular material (Frenklach and Feigelson, 1989; Cherchneff et al., 1992). Those materials make up the major fraction of carbon in space.

\section{FROM THE INTERSTELLAR MEDIUM TO THE TERRESTRIAL PLANETS}

Currently 143 gas-phase molecules, many of them organic, have been identified in interstellar space, and $\sim 60$ molecules have been detected in circumstellar environments (see http://www.astrochemistry.net) (Charnley et al., 2003). Many of the Si-bearing and metal-bearing molecules are only found in circumstellar shells. Apart from silicates, many constituents have been proposed to be present in interstellar grain mantles, including amorphous carbon, hydrogenated amorphous carbon, diamond, refractory organics, and carbonaceous networks such as coal, soot, graphite, quenched-carbonaceous condensates, and others (for reviews, see Henning and Salama, 1998; Ehrenfreund and Charnley, 2000).

\section{Interstellar carbon compounds and their abundance}

PAHs are observed in galactic and extragalactic regions, and represent the most abundant carbonaceous gas-phase molecules in space (Tielens et al., 1999; Yan et al., 2005). Laboratory studies and theoretical calculations have provided important insights into their size and charge state distribution (e.g., Salama et al., 1996; Allamandola et al., 1999; Ruiterkamp et al., 2005b). All three isoforms of carbon-diamond, graphite, and fullerene-have been identified in space environ- ments (Cataldo, 2004). Diamonds were proposed to be the carriers of the 3.4 and $3.5 \mu \mathrm{m}$ emission bands (Guillois et al., 1999) observed in planetary nebulae. Graphite has not been unambiguously identified in the interstellar medium but is present in low abundances in meteorites (Nuth, 1985). The third isoform of carbon is the polyhedral $C_{60}$ fullerene first discussed by Kroto et al. (1985). It has been suggested that fullerenes may be formed in small amounts in envelopes of $\mathrm{R}$ Coronae Borealis stars (Goeres and Sedlmayr, 1992). Rietmeijer et al. (2004) showed that cosmic soot analogs consist of close packed metastable $\mathrm{C}_{60}$ and giant fullerenes. Fingerprints of the $\mathrm{C}_{60}{ }^{+}$ion were discovered in the near-infrared spectra of stars crossing the material of diffuse interstellar clouds (Foing and Ehrenfreund, 1994, 1997). Higher fullerenes have been identified in meteorites (Becker and Bunch, 1997). Laboratory simulations in combination with interstellar observations support the idea that the predominant fraction of carbon in space is present as solid macromolecular carbon (e.g., Pendleton and Allamandola, 2002) or amorphous and hydrogenated amorphous carbon (Mennella et al., 1998; Duley and Lazarev, 2004; Dartois et al., 2005). Fullerenes (Iglesias-Groth, 2004) or defective carbon "onions" (Tomita et al., 2004) have also been proposed.

Cosmic abundances in the interstellar medium are derived by measuring elemental abundances in stellar photospheres, the atmospheric layer just above the stellar surface. Such measurements indicate the amount of elements available for the formation of molecules and particles. Cosmic dust models indicate that up to $80 \%$ of the carbon in the photon-dominated diffuse interstellar medium is incorporated into solid aromatic macromolecules and gaseous PAHs (Mennella et al., 1998; Tielens et al., 1999). CO gas and C-based ice species (such as $\mathrm{CO}, \mathrm{CO}_{2}, \mathrm{CH}_{3} \mathrm{OH}$, and others) may make up to $\sim 25 \%$ of the carbon in cold dense interstellar regions. It must be taken into account that elemental abundances (e.g., of carbon) in stars and the interstellar medium may not be the same. Stellar photospheres may not completely represent the composition of interstellar material from which stars are formed, resulting either from the possible underestimation of the degree of heavy element settling in the stellar atmosphere or from incomplete incorporation of heavy elements in stars during star formation (Li, 2005). However, prebiotic molecules (as used in life as we know it) observed in the interstellar 
medium have negligible abundances compared with aromatic moieties; organic material in space is predominantly macromolecular and aromatic in nature.

\section{Formation of a solar system and extraterrestrial delivery}

Interstellar gas and icy or refractory dust (composed of silicates and carbonaceous material) provide the raw material for the formation of stars and planets. Gravitational collapse of interstellar clouds leads to the formation of a protoplanetary region with a central condensation developing into a star surrounded by a disk (Mannings et al., 2000; Boss, 2004). During the formation of a solar system, interstellar gas and dust are mixed, processed, and partly destroyed according to the distance from the forming star (Chick and Cassen, 1997). Radiation chemistry involving x-rays and UV light may also act on close or upper disk layers. Turbulent motions lead to radial mixing of the products within the disk (Irvine et al., 2000; Ehrenfreund et al., 2004; Markwick and Charnley, 2004). Within disks, dust assembles into grains, which in turn assemble into boloids and ultimately into kilometer-sized planetesimals that interact gravitationally (Weidenschilling and Cuzzi, 1993). The key steps in this assembly process are still a mystery, but once self-gravitation begins planetesimals grow by collision to form terrestrial planets (Blum, 2004).

Many planetesimals formed in such a manner could not be integrated into planets, and their ultimate fate was dependent on their location within the nascent solar system. The giant planets played an important role in stabilizing the structure of the solar system by trapping remnant bodies within defined regions such as the asteroid belt, or by ejecting them into outer solar system regions (Kuiper Belt) and beyond (Oort Cloud). The deflection of small bodies in the inner solar system led to large impacts on the forming terrestrial planets. Comets probably contributed most of the carbonaceous compounds during the heavy bombardment phase 4.5-4 billion years ago (Ehrenfreund et al., 2002). Fragments of asteroids and comets such as interplanetary dust particles and carbonaceous meteorites were probably among the other major extraterrestrial contributors of carbon (Chyba et al., 1990; Chyba and Sagan, 1992; Oró and Lazcano, 1997).

Comets are principally formed in the region be- yond Jupiter. They are predominantly icy bodies that contain some silicates and refractory organic material (Greenberg, 1998). More than 50 molecules have been identified in cometary comae (Crovisier, 2004). Many small organic molecules observed in cometary comae probably originate wholly or partially from the decomposition of larger molecules or particles, which indicates that large polymers such as polyoxymethylene and HCN-polymers may be present in comets (see Ehrenfreund et al., 2004). Carbonaceous meteorites contain a substantial amount of carbon (up to $3 \%$ by weight) and exhibit evidence of thermal and aqueous alteration believed to have occurred on their parent bodies. Up to $90 \%$ of this carbon is macromolecular (Gardinier et al., 2000; Sephton et al., 2000; Cody and Alexander, 2005). The insoluble macromolecular matter in carbonaceous meteorites is composed of mono- and polyaromatic units bonded with oxygen, sulfur, and small aliphatic chains. More than 70 amino acids have been identified in the soluble fraction of such samples in addition to many other organic compounds, including N-heterocycles, carboxylic acids, sulfonic and phosphonic acids, and aliphatic and aromatic hydrocarbons (Cooper et al., 1992; Botta and Bada, 2002; Sephton, 2002). The presence of organic matter in both anhydrous and hydrated interplanetary dust particles has been discussed (Clemett et al., 1993; Flynn et al., 2003).

\section{What material was delivered to the early Earth, and what are the implications for life?}

The large quantities of extraterrestrial material delivered to young terrestrial planetary surfaces in the early history of our solar system may have provided the material necessary for the emergence of life. Tables 2 and 3 summarize the organic material that has been measured to date in comets and meteorites. Data are predominantly compiled from the analysis of the Murchison meteorite and observations of bright comets (e.g., for a review see Ehrenfreund et al., 2002; Crovisier, 2004). Recent bright comets such as Hale-Bopp have been investigated by spectroscopy from the UV to the radio range in unprecedented detail. Though several classes of organic compounds important in contemporary biochemistry appear on the list of meteoritic compounds, the dominant form of carbonaceous material is aromatic. This is similar to the original interstellar cloud material as discussed in The Formation and Evolution of Organic Material in Space and From the Inter- 
Table 2. Production Rates Relative to Water of Organic Molecules in the Coma of Comet C/1995 O1 Hale-Bopp

\begin{tabular}{|c|c|}
\hline Molecule & C/1995 O1 Hale-Bopp \\
\hline $\mathrm{H}_{2} \mathrm{O}$ & 100 \\
\hline $\mathrm{CO}$ & $12-23$ \\
\hline $\mathrm{CO}_{2}$ & 6 \\
\hline $\mathrm{CH}_{4}$ & 1.5 \\
\hline $\mathrm{C}_{2} \mathrm{H}_{2}$ & $0.1-0.3$ \\
\hline $\mathrm{C}_{2} \mathrm{H}_{6}$ & 0.6 \\
\hline $\mathrm{CH}_{3} \mathrm{OH}$ & 2.4 \\
\hline $\mathrm{H}_{2} \mathrm{CO}$ & 1.1 \\
\hline $\mathrm{HCOOH}$ & 0.09 \\
\hline $\mathrm{HCOOCH}_{3}$ & 0.08 \\
\hline $\mathrm{CH}_{3} \mathrm{CHO}$ & 0.02 \\
\hline $\mathrm{NH}_{2} \mathrm{CHO}$ & 0.015 \\
\hline $\mathrm{HCN}$ & 0.25 \\
\hline $\mathrm{HNCO}$ & 0.10 \\
\hline HNC & 0.04 \\
\hline $\mathrm{CH}_{3} \mathrm{CN}$ & 0.02 \\
\hline $\mathrm{HC}_{3} \mathrm{~N}$ & 0.02 \\
\hline OCS & 0.4 \\
\hline $\mathrm{CS}_{2}$ & 0.2 \\
\hline $\mathrm{H}_{2} \mathrm{CS}$ & 0.05 \\
\hline
\end{tabular}

Data are taken from Bockelée-Morvan et al. (2004).

stellar Medium to the Terrestrial Planets. This aromatic material has not been considered useful for biogenesis; however, aromatic macromolecular materials are known to fragment upon heating, oxidation, or hydrolysis into subunits that may have prebiotic roles. The large quantities of aromatic material compared with trace concentrations of amino acids, N-heterocycles, and sugars arriving via extraterrestrial delivery suggest that it might be useful to consider how aromatic material could have contributed to life's origin. The resistance of aromatic compounds to thermal and radiation processing in space is reflected in their high abundance. Consequently, this may apply to the young Earth as well.

\section{TERRESTRIAL SOURCES OF ORGANIC MATTER AND POSSIBLE PREBIOTIC SCENARIOS}

In addition to extraterrestrial sources, endogenous synthesis on Earth from atmospheric reactions, hydrothermal vents, or serpentinization may have contributed to the pool of precursor molecules and complex organics. Atmospheric synthesis of organics has been a favorite scenario for many decades. The primitive atmosphere may, however, have been a minor contributor to the organic inventory on the young Earth if it was not reducing. The limited knowledge of the exact density and composition of the atmosphere and the temperature and radiation conditions on the Earth makes it difficult to estimate the relative contributions of extraterrestrial delivery and endogenous atmospheric synthesis. During heavy impacts in the first 500 million years, the Earth's climate may have alternated between exceedingly hot periods (in which most organic compounds were destroyed) and cold periods, making it difficult for life to become established. The concentration of organic material in the prebiotic environment is of crucial importance. Precursor molecules need to be concentrated to react before being degraded by the environment. Small molecules, crucial for living cells in modern chemistry, such as amino acids, heterocycles, and sugars, are rather fragile compounds with regard to temperature, extremes of $\mathrm{pH}$, and radiation.

Table 3. Abundances and Type of Organic Matter in the Best-Studied Carbonaceous Meteorite, Murchison (CM2)

\begin{tabular}{|c|c|c|}
\hline \multirow[b]{2}{*}{ Compound class } & \multicolumn{2}{|c|}{ Abundances } \\
\hline & $\%$ & ppm \\
\hline Macromolecular material & 1.45 & \\
\hline $\mathrm{CO}_{2}$ & & 106 \\
\hline $\mathrm{CO}$ & & 0.06 \\
\hline $\mathrm{CH}_{4}$ & & 0.14 \\
\hline Amino acids & & 60 \\
\hline Aliphatic hydrocarbons & & $12-35$ \\
\hline Aromatic hydrocarbons & & $15-28$ \\
\hline Fullerenes & & $>1$ \\
\hline Carboxylic acids & & 332 \\
\hline$\alpha$-Hydroxycarboxylic acids & & 15 \\
\hline Dicarboxylic acids & & 26 \\
\hline Pyridinecarboxylic acids & & $>7$ \\
\hline Basic N-heterocycles & & $0.05-0.5$ \\
\hline Pyrimidines (uracil and thymine) & & 0.06 \\
\hline Purines & & 1.2 \\
\hline Benzothiophenes & & 0.3 \\
\hline Dicarboximides & & $>50$ \\
\hline Amines & & 8 \\
\hline \multicolumn{3}{|l|}{ Amides } \\
\hline Linear & & $>70$ \\
\hline Cyclic & & $>2$ \\
\hline Alcohols & & 11 \\
\hline Aldehydes & & 11 \\
\hline Ketones & & 16 \\
\hline Sugar-related compounds & & $\sim 24$ \\
\hline Urea & & 25 \\
\hline
\end{tabular}

Data are compiled from Botta and Bada (2002), Sephton (2002), and Sephton and Botta (2005). 
Therefore, depending on the rates of synthesis, it may be questionable as to whether such compounds could have been involved in the formation of the first living entities.

\section{Early Earth conditions}

The three main requirements for life as we know it are organic compounds, liquid water, and free energy. These may also have been crucial for the establishment of the first living systems. It seems reasonable to assume that life required a solvent for reactions to occur in (water), compounds for the living system to be built from (most likely organic compounds), and free energy to allow for chemical evolution to proceed both by providing the driving force for chemical reactions and for allowing fluctuations in the environment that would provide both energy and a natural selection pressure for the evolution of the system. The early solar system appears to have provided all of these, and it seems possible that these conditions could be present on numerous other bodies in the galaxy. The synthesis of small organic compounds from simple gas mixtures is one of the best experimentally understood steps in the origin of life. The efficiency of organic production and the nature of the products depend on the specific type of energy introduced and the gas mixture used.

There is little agreement on the composition of the primitive atmosphere. Opinion varies from strongly reducing $\left(\mathrm{CH}_{4}+\mathrm{N}_{2}+\mathrm{NH}_{3}+\mathrm{H}_{2} \mathrm{O}\right.$, or
$\left.\mathrm{CO}_{2}+\mathrm{H}_{2}+\mathrm{H}_{2} \mathrm{O}+\mathrm{N}_{2}\right)$ to neutral $\left(\mathrm{CO}_{2}+\mathrm{N}_{2}+\right.$ $\mathrm{H}_{2} \mathrm{O}$ ), though it is generally believed that free $\mathrm{O}_{2}$ was absent (Canuto et al., 1983). There has been less experimental work with gas mixtures that contain $\mathrm{CO}$ or $\mathrm{CO}_{2}$ as carbon source in place of $\mathrm{CH}_{4}$, though $\mathrm{CO}$-dominated atmospheres could not have existed except transiently (Miyakawa et al., 2002a). As mentioned earlier, the efficiency of organic production depends on the reducing nature of the atmosphere; neutral atmospheres may be some $10^{5}$-fold less efficient at producing organics than reducing atmospheres (Miller, 1998). Recently, the possibility that the early atmosphere contained significant amounts of $\mathrm{H}_{2}$ has been resuscitated (Tian et al., 2005).

The early Earth can be viewed as a dynamic system warmed by heat from accretion, the sinking of iron into the core, impacts, and decay of radioactive elements. The most abundant energy sources on Earth today are light, electric discharges from lightning and static electricity, radioactive decay, and volcanism (Table 4). Energy fluxes from these sources may have been considerably different in the primitive environment. For example, the primitive Sun would have provided a much higher flux of UV radiation, though the total luminosity was lower. It is likely that volcanic activity was more intense and energy from radioactive decay was more abundant (Mosqueira et al., 1996). Shock waves from extraterrestrial impactors and thunder were also probably more common during the planetary accretion process. It is difficult to es-

Table 4. Fluxes of Energy from Various Sources on the Present Earth: Solar Fluxes at Sea Level

\begin{tabular}{lcr}
\hline Source & Energy $\left(\right.$ cal/cm $^{2} /$ year $)$ & Energy $\left(\mathrm{J} / \mathrm{cm}^{2} /\right.$ year $)$ \\
\hline Total radiation from Sun & 260,000 & $1,090,000$ \\
UV light & & 14,000 \\
$<300 \mathrm{~nm}$ & 3,400 & 2360 \\
$<250 \mathrm{~nm}$ & 563 & 170 \\
$<200 \mathrm{~nm}$ & 41 & 7 \\
$<150 \mathrm{~nm}$ & 1.7 & 17 \\
Electric discharges & $4.0^{\mathrm{a}}$ & 0.006 \\
Cosmic rays & 0.0015 & 3.0 \\
Radioactivity (to $1.0 \mathrm{~km})$ & 0.8 & 0.5 \\
Volcanoes & 0.13 & 4.6 \\
Shock waves & $1.1^{\mathrm{b}}$ & 4.6 \\
\hline
\end{tabular}

Fluxes on the primitive Earth may have differed, in particular, the flux of shorter wavelength UV light, especially if there was increased UV output by the early Sun, and in the absence of ozone and molecular oxygen in the primitive atmosphere. The majority of the solar flux incident on the upper atmosphere is presently in the form of longer wavelength radiation $(\lambda>300 \mathrm{~nm})$ of lower energy per photon, which is unable to engage in useful atmospheric prebiotic chemistry. Adapted from Miller and Orgel (1974).

a $3 \mathrm{cal} / \mathrm{cm}^{2} /$ year of corona discharge and $1 \mathrm{cal} / \mathrm{cm}^{2} /$ year of lightning.

$\mathrm{b} 1 \mathrm{cal} / \mathrm{cm}^{2} /$ year of this is the shock wave of lightning bolts and is also included under electric discharges. 
timate the difference in electric discharge flux early in the Earth's history.

The Sun is expected to have followed a typical stellar evolution for its mass and spectral type (G2 class). It is expected that solar luminosity would have been $\sim 30 \%$ less around the time of the origin of life (Kasting and Catling, 2003). A possible consequence of this is that the prebiotic Earth may have frozen completely to a depth of $\sim 300 \mathrm{~m}$ (Bada et al., 1994), though there is evidence that liquid water was present $\sim 4$ billion years ago (Mojzsis et al., 2001; Wilde et al., 2001). The presence of liquid surface water would have required that the early Earth maintained a heat balance that offset the lower solar flux from the faint young Sun. Atmospheric greenhouse warming from $\mathrm{CH}_{4}, \mathrm{NH}_{3}$, or $\mathrm{CO}_{2}$ has been proposed, but this is still debated (Kasting and Catling, 2003).

Prebiotic synthesis of amino acids, nucleobases, and sugars

The first successful amino acid synthesis under prebiotic conditions was carried out with an electric discharge and a strongly reducing model atmosphere of $\mathrm{CH}_{4}, \mathrm{NH}_{3}, \mathrm{H}_{2} \mathrm{O}$, and $\mathrm{H}_{2}$ (Miller, 1953). This experiment produced a large yield of racemic amino acids, together with hydroxy acids, short aliphatic acids, and urea. The products obtained give clues to the mechanism of synthesis. The yield of both $\alpha$-amino and $\alpha$-hydroxy acids of the same carbon skeletons suggested that the compounds were formed by the Strecker amino acid and cyanohydrin hydroxy acid syntheses. These reactions occur when aldehydes or ketones, ammonia, and cyanide (derived from gas-phase reactions of $\mathrm{CH}_{4}, \mathrm{NH}_{3}, \mathrm{H}_{2} \mathrm{O}$, and $\mathrm{H}_{2}$ ) are allowed to react in water. Detailed studies of the equilibrium and rate constants of these reactions have been performed (Miller, 1957). The results demonstrate that both amino and hydroxy acids can be synthesized at high dilutions of HCN and aldehydes in a simulated primitive ocean. The reaction rates depend on temperature, $\mathrm{pH}$, and reactant concentrations, but are rapid on a geologic time scale. It was determined that such reactions could occur starting from even extremely low concentrations of precursors, as might be delivered to the oceans and even from relatively low-yielding atmospheric synthesis in neutral $\mathrm{CO}_{2} / \mathrm{N}_{2}$ atmospheres (Schlesinger and Miller, 1973, 1983).

The Strecker synthesis of amino acids requires the presence of $\mathrm{NH}_{3}$ in the prebiotic environment.
Gaseous $\mathrm{NH}_{3}$ is rapidly decomposed by ultraviolet light (Kuhn and Atreya, 1979), and during early Archean times the absence of a significant ozone layer would have imposed an upper limit to its atmospheric concentration. Since $\mathrm{NH}_{3}$ is soluble in water, most of the Earth's $\mathrm{NH}_{3}$, if the primitive oceans and sediments were buffered to the modern value of $\mathrm{pH} \sim 8$, would have been present as dissolved $\mathrm{NH}_{4}{ }^{+}$(the $\mathrm{pK}$ a of $\mathrm{NH}_{3}$ is 9.2) in equilibrium with dissolved $\mathrm{NH}_{3}$.

Nucleic acids are the central repository of the information organisms use to construct enzymes via protein synthesis. One of the principal characteristics of life is the ability to transfer information from one generation to the next. Nucleic acids seem uniquely suited for this function, and thus a considerable amount of attention has been dedicated to elucidate their prebiotic synthesis. The first evidence that the components of nucleic acids could have been synthesized non-biologically was provided in 1960 when it was found that concentrated solutions of ammonium cyanide refluxed for a few days produced adenine (Oró, 1960; Oró and Kimball, 1961). Other purines, including guanine, hypoxanthine, xanthine, and diaminopurine, have since been produced using variations of this synthesis (Sanchez et al., 1968). The kinetics of the intermediate reactions in this synthesis have been used to delineate the limits of geochemically plausible synthesis. The steady-state concentrations of $\mathrm{HCN}$ would have depended on the $\mathrm{pH}$, the temperature of the early oceans, and the input rate of $\mathrm{HCN}$ from atmospheric synthesis (itself depending on the reducing nature of the atmosphere) or extraterrestrial delivery. Assuming favorable production rates, steady-state concentrations of $\mathrm{HCN}$ of $2 \times 10^{-6} \mathrm{M}$ at $\mathrm{pH} 8$ and $0^{\circ} \mathrm{C}$ in the primitive oceans were estimated (Miyakawa et al., 2002b). At $100^{\circ} \mathrm{C}$ and $\mathrm{pH} \sim 8$ the steady-state concentration was estimated as $7 \times 10^{-13} \mathrm{M}$. Oligomerization and hydrolysis compete at approximately $10^{-2} \mathrm{M}$ concentrations of HCN at pH 9 (Sanchez et al., 1966a), though it has been shown that adenine is still produced from solutions as dilute as $10^{-3} \mathrm{M}$ (Miyakawa et al., 2002c). Clearly, some concentration mechanism would have been necessary, with eutectic freezing seemingly the most likely, as $\mathrm{HCN}$ cannot be concentrated by evaporation (Sanchez et al., 1966a).

The prebiotic synthesis of pyrimidines has also been investigated. Cytosine and uracil are synthesized from the reaction of aqueous cyanoacetylene and cyanate (Sanchez et al., 1966b), both 
of which are produced in electric discharge reactions. Later it was found that cyanoacetaldehyde (produced from cyanoacetylene) and urea react to form cytosine and uracil in high yield when dried together (Robertson and Miller, 1995). The reaction of uracil with formaldehyde and formate gives thymine in good yield (Choughuley et al., 1977).

Most biological sugars have the empirical formula $\left(\mathrm{CH}_{2} \mathrm{O}\right)_{n}$, a point underscored by Butlerov's (1861) discovery of the formose reaction, which showed that a complex mixture of biologically important sugars could be formed by the reaction of $\mathrm{HCHO}$ under basic conditions. The mechanism of the Butlerov synthesis is complex and incompletely understood. It depends on the presence of suitable inorganic catalysts; in the absence of basic catalysts, little or no sugar is obtained. There are three major obstacles to the relevance of the formose reaction as a source of sugars on the primitive Earth. The first problem is that the Butlerov synthesis gives a wide variety of straightchain and branched sugars. The second problem is that the conditions of synthesis are also conducive to the degradation of sugars (Reid and Orgel, 1967). Sugars undergo various irreversible degradation reactions on geologically short time scales, which would have prohibited their accumulation on the primitive Earth. At $\mathrm{pH} \sim 7$, the half-life for decomposition of ribose is $73 \mathrm{~min}$ at $100^{\circ} \mathrm{C}$, and 44 years at $0^{\circ} \mathrm{C}$ (Larralde et al., 1995). The same is true for most other sugars. The third problem is that the concentrations of HCHO required appear to be prebiotically implausible (Pinto et al., 1980).

Amphiphilic molecules are especially interesting because of their ability under appropriate conditions of $\mathrm{pH}$, temperature, and concentration to assemble spontaneously into micelles, vesicles, and other types of aggregates (Segré et al., 2001). The origin of life picture proposed in the "Lipid World" was originally developed by Luisi et al. (1988) and by Morowitz et al. (1988), who worked toward assembling protocells based on a self-reproducing lipid vesicle encapsulating a self-replicating RNA protogene. Simple fatty acids are found in meteorites and were probably available at the time of the origin of life. Encapsulation of self-assembling structures, such as microtubules, within liposomes has also served as an incomplete attempt to develop a protocell model (Hotani et al., 1992). An interesting twist to the Lipid World came recently with the proposed role of atmospheric aerosols in the origins of life (Tuck, 2002).

Most prebiotic simulations do not generate large amounts of fatty acids, with the exception of certain hydrothermal vent simulations, which arguably use unreasonably high concentrations of reactants (McCollom et al., 1999). The Murchison meteorite contains small amounts of highermolecular-weight straight-chain fatty acids, some of which may be contamination (Yuen and Kvenvolden, 1973). Amphiphilic components have been positively identified in the Murchison meteorite (Deamer, 1985). It should be noted that phospholipids can also be synthesized fairly readily from simple prebiotic starting materials under simulated drying beach conditions (see Segré et al., 2001, and references therein).

Energy-transducing molecules have not been as well investigated, if only because "metabolismfirst" origins of life theories remain less well elucidated (Smith and Morowitz, 2004). However, a number of potential electron carrier molecules and photon-harvesting molecules have been reported in prebiotic simulations. Notable among these are porphyrin analogues (Hodgson and Ponnamperuma, 1968; Simionescu et al., 1980; Chadha and Choughuley, 1984) and PAHs (Deamer, 1992). As yet, these have not been demonstrated to be useful prebiotically; however, several schemes have been suggested (Stilwell, 1977; Stephan, 2002).

\section{Informational polymers}

One popular theory for the origin of life proposes the existence of an RNA World, a time when RNA molecules played the role of both catalysts and genetic molecule in biochemistry (Gesteland et al., 1999; Joyce, 2002). A great deal of research has been carried out on the prebiotic synthesis of nucleosides and nucleotides. While considerable progress has been made in this area, a number of chemically implausible steps in this scheme still render it problematic. This raises the intriguing possibility that the first informational polymer may not have been RNA-based, which will be explored in more detail herein. The most promising nucleoside syntheses start from purines and pure D-ribose in drying reactions, which simulate conditions that might occur in an evaporating basin (Fuller et al., 1972). Using hypoxanthine and a mixture of salts reminiscent of those found in seawater, up to $8 \%$ of $\alpha$-D-inosine 
is formed, along with the $\beta$-isomer. Adenine and guanine give lower yields, and in both cases a mixture of $\alpha$ - and $\beta$-isomers are obtained (Fuller et al., 1972). Pyrimidine nucleosides have proven to be much more difficult to synthesize. Direct heating of ribose and uracil or cytosine has thus far failed to produce uridine or cytidine. Pyrimidine nucleosides have, however, been produced by more complicated multistep schemes involving photochemical rearrangement (Sanchez and Orgel, 1970; Ingar et al., 2003).

The difficulties with prebiotic ribose synthesis and nucleoside formation have led some to speculate that perhaps a simpler genetic molecule with a more robust prebiotic synthesis preceded RNA. A number of alternatives have been investigated (Fig. 2). Some substitute other sugars for ribose (Beier et al., 1999; Eschenmoser, 2004), including the structurally very simple threose nucleic acid (TNA). These molecules would likely suffer from the same drawbacks as RNA, which include the difficulty of selective sugar synthesis, sugar instability, and the difficulty of nucleoside formation. More exotic nucleoside alternatives have been proposed based on the peptide nucleic acid (PNA) analogues (Nielsen et al., 1991) and other acyclic monomers (Joyce et al., 1987). Miller and co-workers (Nelson et al., 2000) were able to demonstrate the synthesis of the components of PNA under the same conditions required for the synthesis of the biological purines and pyrimidines. The assembly of the molecules into oligomers has not yet been demonstrated and may be unlikely (Eriksson et al., 1998). There may be alternative structures that have not yet been investigated but may sidestep some of the prob- lems with the PNA backbone. As of this writing, the simplest acyclic nucleoside analogue, glycol nucleic acid (GNA), has been shown to form stable Watson-Crick base-paired structures (Zhang et al., 2005) (see Fig. 2 for examples of some proposed alternative nucleic acid structures).

\section{Phosphates}

Condensed phosphates, principally in the form of ATP, are the universal biological energy currency. However, abiological dehydration reactions are extremely difficult in aqueous solution because of high water activity, and it has been suggested that condensed phosphates are unlikely prebiotic compounds (Keefe and Miller, 1995). There is the intriguing possibility, however, that phosphates may have entered early biochemistry in the form of phosphonic acids (De Graaf and Schwartz, 2005), which are present in the Murchison meteorite (Cooper et al., 1992). There is some evidence that condensed phosphates are emitted in volcanic fumaroles (Yamagata et al., 1991). The heating of ammonium phosphates with urea leads to a mixture of highmolecular-weight polyphosphates (Osterberg and Orgel, 1972). Although polyphosphates are not especially good phosphorylating reagents under prebiotic conditions, they tend to degrade, especially in the presence of divalent cations at high temperatures, to cyclic phosphates such as trimetaphosphate. Trimetaphosphate has been shown to be a phosphorylating agent for various prebiological molecules, including amino acids and nucleosides (Schwartz, 1969; Rabinowitz and Hampai, 1978). Another intriguing possibility is

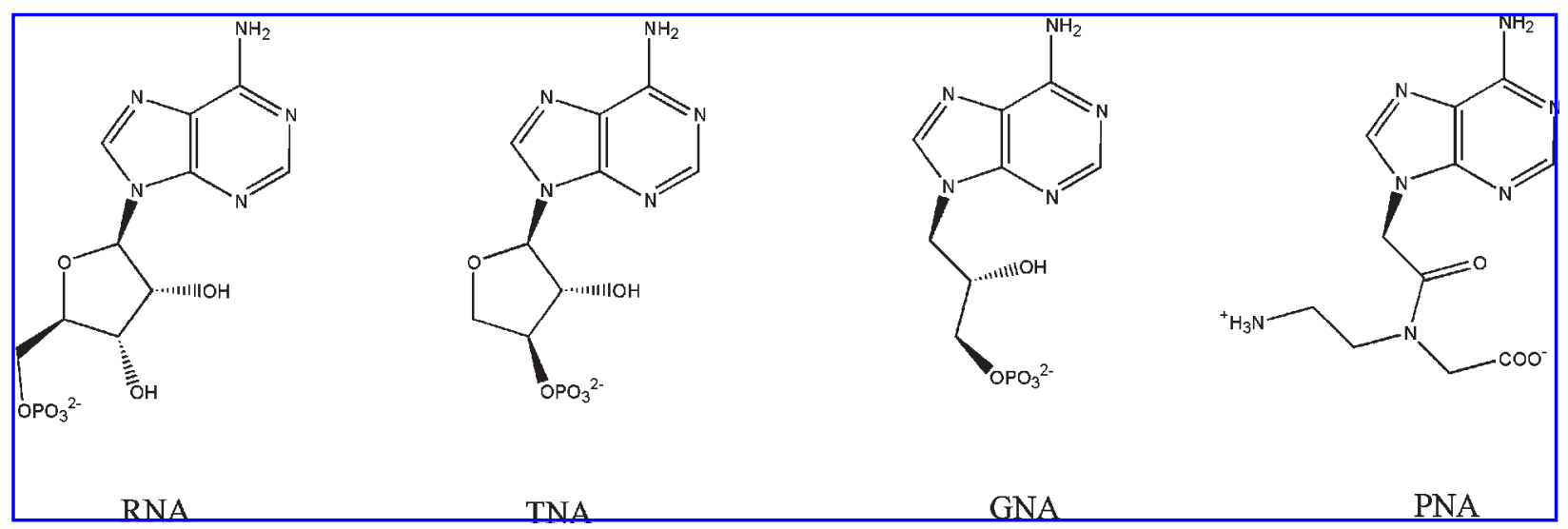

FIG. 2. Some possible monomers that could have been used to construct a primordial genetic molecule. 
that reactive phosphates were delivered extraterrestrially in the form of phosphide minerals (Pasek and Lauretta, 2005).

Nucleosides can also be phosphorylated with acidic phosphates such as $\mathrm{NaH}_{2} \mathrm{PO}_{4}$ when dryheated (Beck et al., 1967) and by heating with ammonium phosphate and urea at moderate temperatures, as might occur in a drying basin (Lohrmann and Orgel, 1971). Yields as high as $20 \%$ of mixed nucleotide monophosphates were achieved by heating nucleosides with the mineral hydroxyapatite, urea, and ammonium phosphate (Lohrmann and Orgel, 1971). Interestingly, nucleoside $2^{\prime}, 3^{\prime}$-cyclic phosphates are major products of such syntheses. These have been shown to be polymerizable into oligonucleotides under drying conditions (Verlander et al., 1973).

\section{Subsurface synthesis}

The discovery of hydrothermal vents at the oceanic ridge crests was a major oceanographic discovery (Corliss et al., 1979). A hot microbial biosphere associated with active volcanism along the global mid-ocean ridge network has improved our knowledge of life adapting to extreme environments (Kelley et al., 2002). Since hydrothermal circulation probably began early in the Earth's history, it is likely that vents were present in the Archean oceans. It has been suggested that organic compounds are produced during passage through the temperature gradient of the $350^{\circ} \mathrm{C}$ vent waters to the $0^{\circ} \mathrm{C}$ ambient ocean waters under high pressure (Corliss et al., 1981). Polymerization of the organic compounds thus formed, followed by their self-organization, has also been proposed to take place in these environments. At first glance, submarine hydrothermal springs appear to be ideally suited for creating life, given the geological plausibility of a hot early Earth. Unfortunately, it is difficult to corroborate these ideas with the composition of modern vent effluent, as most of the organic material released from modern sources is degraded biological material, and it is difficult to separate the biotic from the abiotic (nonbiological) components of these reactions (Miller and Bada, 1988; Ferris, 1992; Shock and Schulte, 1998; Holm and Charlou, 2001).

Iron sulfides have been proposed to play a potential catalytic role in the formation of organometallic compounds (Cody et al., 2000) and in the origin of life (Russell and Hall, 1997;
Russell and Martin, 2004). In the latter case, though, hydrothermal solutions are buffered at lower temperatures $\left(<115^{\circ} \mathrm{C}\right)$ and higher $\mathrm{pH}$ (10-11). The most elaborate articulation of hydrothermal vent biopoiesis stems from the work of Wächtershäuser (1988), who has argued that life began with the appearance of an autocatalytic, two-dimensional chemolithtrophic metabolic system based on molecular hydrogen and a reduction potential provided by the formation of the highly insoluble mineral pyrite $\left(\mathrm{FeS}_{2}\right)$ :

$$
\begin{array}{r}
\mathrm{FeS}+\mathrm{H}_{2} \mathrm{~S} \rightarrow \mathrm{FeS}_{2}+\mathrm{H}_{2}\left(E^{\circ}=620 \mathrm{mV}, \Delta G^{\circ}=\right. \\
-9.23 \mathrm{kcal} / \mathrm{mol})
\end{array}
$$

The FeS/ $\mathrm{H}_{2} \mathrm{~S}$ combination is a strong reductant for a variety of organic compounds under mild conditions. The FeS $/ \mathrm{H}_{2} \mathrm{~S}$ system has not been shown to reduce $\mathrm{CO}_{2}$ to amino acids, purines, or pyrimidines in the laboratory, though there is more than adequate free energy to do so (Keefe et al., 1995). However, pyrite formation can produce molecular hydrogen and reduce nitrate to ammonia, and acetylene to ethylene (Maden, 1995). Although speculative, it is possible that under certain as yet unspecified geological conditions the $\mathrm{FeS} / \mathrm{H}_{2} \mathrm{~S}$ combination could have reduced $\mathrm{CO}$ and $\mathrm{CO}_{2}$ released from deep-sea vents, which would have led to biochemical monomers such as amino acids and nucleosides (Orgel, 1998). Peptide synthesis could have taken place in an iron and nickel sulfide system (Huber and Wächtershäuser, 1998) involving amino acids formed by electric discharges via a Strecker-type synthesis, though this scenario might require the transportation of compounds formed in other environments to the deep-sea vents (Rode, 1999). Environmental concentrations of reactants may, however, be prohibitively low for such polymerization reactions.

Another reaction pathway that is sometimes discussed in the context of hydrothermal vents is the Fischer-Tropsch type (FTT) synthesis (Ferris, 1992). This reaction has also been proposed as a synthetic mechanism in volcanoes and meteorites as well as on the surface of extraterrestrial mineral grains that enter the Earth's atmosphere (Hayatsu et al., 1971, 1972; Basiuk and NavarroGonzalez, 1996; Hill and Nuth, 2003). The reaction involves the passage of gases over hot iron, nickel, or silicate catalysts, whereby small organic molecules are generated via surface catalysis. Although the reaction produces many important 
biogenic molecules, the reaction mechanisms are poorly understood. While FTT synthesis is an important industrial process, its prebiotic relevance is unclear. The catalysts used in industry are easily poisoned by sulfide, which is a common component of hydrothermal fluid. The concentrations of gases encountered in vent fluids and the high water activities encountered there may render FTT synthesis difficult. To date, it remains controversial as to whether organic molecules are generated abiotically from oxidized carbon in vent environments.

Serpentinization of oceanic crust is another mechanism by which FTT syntheses are proposed to occur (Holm and Andersson, 1998; Sleep et al., 2004). Conversion of ultramafic peridotite (olivine and pyroxene) to serpentine and magnetite leads to the formation of $\mathrm{H}_{2}$, which can reduce $\mathrm{CO}_{2}$ to $\mathrm{CH}_{4}$. Magnetite has been shown to convert $\mathrm{CO}_{2} / \mathrm{H}_{2}$ to organic compounds under conditions reminiscent of serpentinization (Berndt et al., 1996). Although serpentinization may have resulted in the formation of hydrocarbons in some special subsurface environments, the temperature conditions under which these syntheses occur would not allow the survival of most biogenic compounds. Serpentinization may, however, also have served as a source of $\mathrm{CH}_{4}$ and $\mathrm{H}_{2}$ for atmospheric reactions.

If mineral assemblages in the cooler zones of hydrothermal vent environments were sufficiently reducing, then similar reactions in vent systems may have been a source of atmospheric $\mathrm{H}_{2}, \mathrm{CH}_{4}$, and $\mathrm{NH}_{3}$ (Shock et al., 1998; Kelley et al., 2002, 2005).

In general, organic compounds are more readily decomposed than created at hydrothermal vent temperatures, though this would not apply to the low temperature off-ridge vents (e.g., Russell and Martin, 2004). Most biological molecules have half-lives to hydrolysis on the order of minutes to seconds at the high temperatures associated with hydrothermal vents (Table 5). As noted earlier, ribose and other sugars are extremely thermolabile (Larralde et al., 1995). Pyrimidines, purines, amino acids, nucleotides, and peptides are nearly as labile (White, 1984; Shapiro, 1995). The half-lives for polymers are even shorter as there are many potential breakage points. However, it is possible that hydrothermal vents may serve as synthesis sites for simpler compounds such as acetate (Russell and Martin, 2004) or more complex organic compounds such as fatty acids
Table 5. Half-Lives for the Decomposition OF SOME IMPORTANT Biomolecules AT PH 7 AND VARIOUS TEMPERATURES

\begin{tabular}{lcc}
\hline \multirow{2}{*}{ Compound } & \multicolumn{2}{c}{$\mathrm{t}_{1 / 2}$ (years) at } \\
\cline { 2 - 3 } & $0^{\circ} \mathrm{C}$ & $100^{\circ} \mathrm{C}$ \\
\hline Amino acids & $10^{6}-10^{10}$ & $1-10^{4}$ \\
Sugars (ribose) $^{\mathrm{b}}$ & 44 & $73 \mathrm{~min}$ \\
Nucleobases (ACGTU) $^{\mathrm{c}}$ & $10^{4}-10^{9}$ & $0.05-56$ \\
Nucleosides (uridine) $^{\mathrm{d}}$ & $10^{6}$ & 400 \\
RNA scission $^{\mathrm{d}}$ & 900 & 0.01 \\
DNA depurination $^{\mathrm{d}}$ & $10^{5}$ & 0.06 \\
Peptide hydrolysis $^{\mathrm{d}, \mathrm{e}}$ & $10^{8}$ & $\sim 1$ \\
\hline
\end{tabular}

References: a Miller and Orgel (1974); ${ }^{b}$ Larralde et al. (1995); ' Levy and Miller (1998); ' ${ }^{\mathrm{d}}$ White (1984); ${ }^{2}$ Snider and Wolfenden (2000).

(Shock et al., 1998). The adsorption equilibria of most organics with mineral surfaces tend to be higher at lower temperatures; thus adsorption on mineral surfaces would tend to concentrate any organics created at hydrothermal vents in cooler zones (Sowerby et al., 2001).

The concentrations of biomolecules that could have accumulated on the primitive Earth are governed largely by the rates of production and destruction. Although it is presently not possible to state which compounds were essential for the origin of life, it does seem possible to preclude high temperature environments even if simple organic compounds used in modern biochemistry were involved (Cleaves and Chalmers, 2004).

Much of the upper layers of the Earth's crust are porous. Rock and sediment contain pore spaces between grains, within fractures, or in cavities (Gold, 1999). Both extraterrestrially and atmospherically delivered organic compounds, and in particular subsurface-synthesized organic compounds, might be expected to exist in the pore space within the upper layers of the planetary crust. Water, organic compounds, richly varied catalytic minerals, and free chemical redox energy are all expected to be found within pore spaces. The amount of pore space on a young planet would have been an immense volume and has been proposed as a candidate site for the origin of life (Colgate et al., 2003).

Although significant advances have occurred as a result of more than 50 years of research in prebiotic chemistry using compounds crucial in modern biochemistry, there have been no major breakthroughs in the understanding of the origin of life. Furthermore, early Earth conditions may 
have been extremely variable and hostile. This raises the questions of whether fragile species such as amino acids, nucleobases, and sugars would have been abundant enough to have been useful for the origin of life.

\section{FUNCTIONAL ASSEMBLIES AND THEIR INTEGRATION INTO A MINIMAL LIFE FORM}

Primitive life could, in principle, be based on a radically different chemistry and organizational structure than those of contemporary life. Such alternative scenarios are not discussed here, though they are actively pursued in the artificial life research communities both in simulations and as the foundation for robotics design (Brooks, 2001). In the context of the origins of life, it is not obvious which alternative chemistries or organizational principles should be investigated. It is possible that life based on alternative chemical and organizational principles evolved into life as we know it. For example, clay and other inorganic and organic compounds could have played a key role both as precursors and as catalysts (CairnsSmith, 2005).

As discussed in From the Interstellar Medium to the Terrestrial Planets and in Terrestrial Sources of Organic Matter and Possible Prebiotic Scenarios, small molecules, such as amino acids and N-heterocycles, are easily degraded by temperatures much above $50^{\circ} \mathrm{C}$ and UV radiation (Tables 5 and 6), whereas larger aromatic structures can resist far higher doses of both. Amino acids have very short half-lives (Peeters et al., 2003), and N-heterocycles are more easily destroyed than their carbonaceous cognate molecules such as benzene (Table 6). Heterocycles that contain several $\mathrm{N}$-atoms in the ring, such as adenine, have decreased half-lives when exposed to UV radiation (Peeters et al., 2003, 2005).

As stated earlier, the abundant carbonaceous macromolecular material predominant in space environments consists mainly of aromatic units connected by aliphatic bridges. Benzene $\left(\mathrm{C}_{6} \mathrm{H}_{6}\right)$, the most simple aromatic hydrocarbon, is unreactive to common double bond transformation and, when forced to react (by higher temperature and/or a catalyst), undergoes substitution reactions rather then addition reactions. The enhanced stability (also called aromatic stabilization) of benzene is $36 \mathrm{kcal} / \mathrm{mol}$. PAHs are fused benzene rings, and in particular large catacondensed $\mathrm{PAHs}$ (with decreased $\mathrm{H} / \mathrm{C}$ ratio) are unusually stable to chemical modification. Aromatic units would therefore not, in particular, be rapidly destroyed by temperature and radiation when incorporated in a three-dimensional macromolecule. The structural similarity among the aromatic fractions of the insoluble matter of carbonaceous meteorites indicates that these aromatics were incorporated at an early stage and remained stable despite low temperature hydrothermal reactions (Cody and Alexander, 2005). Chemical oxidation of such macromolecules may yield soluble organic acids. Laboratory studies investigating kerogen-like material confirm an increase in aromaticity upon pyrolysis under vacuum (Ehrenfreund et al., 1991). Infrared spectroscopy has shown that, upon processing kerogens, the more fragile aliphatic bonds decrease and the aromatic $(\mathrm{C}-\mathrm{C})$ ring bonds become predominant.

\section{Functional requirements for early life}

It is assumed that life was initially simpler than, yet functionally similar to, life as we know it today.

Table 6. UV Stability of Heterocycles Measured in the Laboratory in Ar Matrix

\begin{tabular}{lcccc}
\hline & \multicolumn{3}{c}{ Half-life } \\
\cline { 2 - 5 } Species & Lab (s) & DISM (years) & DC $\left(\times 10^{6}\right.$ years $)$ & 1 AU (min) \\
\hline Benzene & 220 & 32 & 3.2 & 54 \\
Pyridine & 123 & 18 & 1.8 & 32 \\
Pyrimidine & 56 & 8 & 0.8 & 14 \\
s-Triazine & 17 & 2.5 & 0.2 & 4 \\
\hline
\end{tabular}

Half-lives were calculated from the laboratory data of Peeters et al. (2005) and extrapolated to diffuse interstellar environment (DISM), dense clouds (DC), and the solar system at 1 AU from the Sun using the appropriate UV fluxes: $1 \times 10^{8}$ photons $/ \mathrm{cm}^{2} / \mathrm{s}$ for the DISM (Mathis et al., 1983), $1 \times 10^{3}$ photons $/ \mathrm{cm}^{2} / \mathrm{s}$ for DC (Prasad and Tarafdar, 1983), and $3 \times 10^{13}$ photons $/ \mathrm{cm}^{2} / \mathrm{s}$ for the solar UV flux $>6 \mathrm{eV}$ at 1 AU distance from the Sun. 


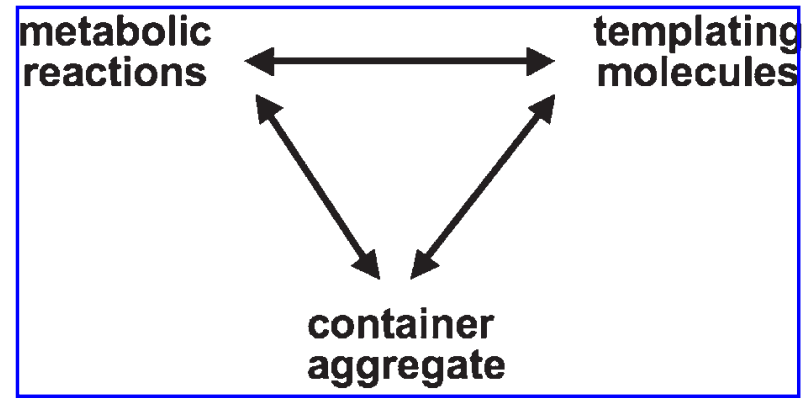

FIG. 3. A simple molecular system could be considered alive if it converts resources into building blocks, grows, replicates, and evolves. Thus a minimal protocell consists of a cooperative structure that combines three functionalities: (1) a metabolism that can convert resources into building blocks, (2) a set of inheritable (templating) molecules that influence the overall growth of the protocell, and (3) a container that keeps (1) and (2) together and acts as a physical structure that facilitates the necessary chemistry for self-reproduction.

A simple molecular system could be considered alive if it turns resources into its own building blocks, grows, replicates, and evolves. This assumes that primitive life would have, at one point, consisted of a compartmentalized genetic system coupled with an energy-harvesting process. While it is difficult to define minimal life, this operational definition is based on three functionally interconnected processes: a metabolic mechanism, a genetic mechanism, and a method of keeping these together through encapsulation (Fig. 3). The metabolic process harnesses energy necessary to convert resource materials into building blocks. The living structure uses the building blocks to grow and replicate. The inheritable molecules influence the growth processes, and as replication proceeds, with some copying error, the functional abilities of the system also change. The accumulated functional differences define the selective advantage of the structures, which enables evolution. The question is, which prebiotically available molecules could have served as container, metabolic, and genetic molecules?

The original question of how prebiotic building blocks can assemble and transform themselves into a minimal living system can now be broken down into two coupled questions:

1. What prebiotic resources can potentially form building blocks, and how can they subsequently be transformed into containers, metabolic reaction networks, and informational polymers?

2. How can these three components assemble into the cooperative organization of a protocell that satisfies the minimal requirements for a living system?

From the astronomical point of view, solid aromatic material is likely to be the most abundant. Aromatic material may be suited to form container compounds, could act as a mediator in metabolic pathways, and could potentially be assembled into informational polymers (Fig. 4 shows typical PAH structures). Table 7 summarizes organic matter identified in the carbona-
FIG. 4. Examples of typical PAH structures and compounds discussed in Functional Assemblies and Their Integration into a Minimal Life Form.

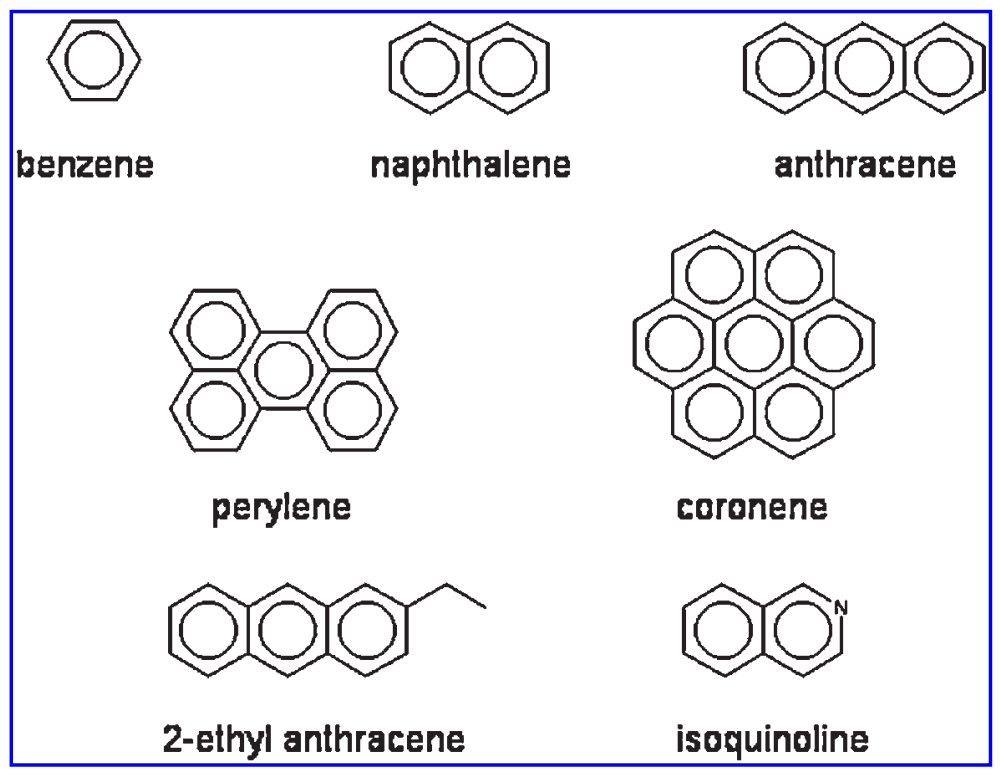


Table 7. Proposed Chemical Building Blocks Based on the Reported Finding from Table 3

\begin{tabular}{|c|c|c|c|}
\hline Prebiotic resources & Building blocks & $\begin{array}{l}\text { Transformed } \\
\text { structures }\end{array}$ & Possible role in a protocell \\
\hline $\begin{array}{l}\text { Carboxylic acids, } \\
\text { amine salts, and } \\
\text { charged PAH }\end{array}$ & Lipid aggregates & $\begin{array}{l}\text { Micelles, vesicles, } \\
\text { bilayer, etc. }\end{array}$ & Container \\
\hline $\begin{array}{l}\text { Aliphatic } \\
\text { hydrocarbons, } \\
\text { carboxylic acids }\end{array}$ & Block co-polymer & $\begin{array}{l}\text { Giant micelles, } \\
\text { vesicles }\end{array}$ & Container \\
\hline $\begin{array}{l}\text { Aromatic } \\
\text { hydrocarbons }\end{array}$ & $\begin{array}{l}\text { Polyaromatic } \\
\text { compounds }\end{array}$ & $\begin{array}{l}\text { Liquid crystal } \\
\text { phase, } \\
\text { calixarenes, etc. }\end{array}$ & $\begin{array}{l}\text { Informational polymer, } \\
\text { metabolic network } \\
\text { components as energy } \\
\text { transducer and inceptor }\end{array}$ \\
\hline $\begin{array}{l}\text { Amides, } \\
\text { dicarboxamides, } \\
\text { amines, carboxylic } \\
\text { acids }\end{array}$ & $\begin{array}{l}\text { Polyamides, PNA, } \\
\text { etc. }\end{array}$ & $\begin{array}{l}\text { double strands, } \\
\text { self-assembling } \\
\text { of peptide }\end{array}$ & $\begin{array}{l}\text { Informational polymer, } \\
\text { metabolic network } \\
\text { components as energy } \\
\text { transducer and inceptor }\end{array}$ \\
\hline $\begin{array}{l}\text { Sugar-related } \\
\text { compounds }\end{array}$ & Oligosaccharides & $\begin{array}{l}\text { Vesicles, } \\
\text { cyclodextrins }\end{array}$ & Container \\
\hline Fullerenes & $\begin{array}{l}\text { Fullerene } \\
\text { complexes }\end{array}$ & & $\begin{array}{l}\text { Metabolic network } \\
\text { components as energy } \\
\text { transducer }\end{array}$ \\
\hline
\end{tabular}

Each molecular component is investigated for its potential functional properties as container components, genetic elements, or metabolic elements.

ceous meteorite Murchison (Table 3) as potential prebiotic resources, their corresponding building blocks, structures that can be transformed, and their possible roles in a protocell. We will first discuss the details of transformed structures, which can potentially contribute to each function (i.e., container, metabolic compound or informational polymer), and then discuss their possible integration into a living system.

\section{Possible container chemistry}

The search for plausible PAH-like molecules that can act as container building blocks is similar, in spirit, to the search for factors that led to the compartmentalization of lipids, a key step in the evolution of life (Luisi et al., 1998; Szostak et al., 2001; Apel et al., 2002; Monnard and Deamer, 2002). Noncovalent protocellular assemblies, generated by catalyzed recruitment of diverse amphiphilic and hydrophobic compounds, could even have constituted the first systems capable of information storage, inheritance, and selection (Segré and Lancet, 2000). Containers can be formed by noncovalent self-assembly. Self-assembly usually requires asymmetric building blocks in the sense that different parts of the building blocks interact differently with the solvent, which is typically water. All bilayer-form- ing molecules are amphiphiles, with a hydrophilic "head" and a hydrophobic "tail" on the same molecule. Because it is unlikely that complex lipid biosynthesis pathways were present in the earliest forms of life, it seems reasonable that the protocell used lipid-like molecules available in the environment. As noted earlier, PAH derivatives, both free and in the form of kerogenlike polymer, represent over $90 \%$ of the organic material of carbonaceous meteorites (see From the Interstellar Medium to the Terrestrial Planets). If there was substantial survival of the organic content of meteoritic and cometary infall during late accretion of the Earth, aromatic molecules would presumably be major components of the organic inventory. Indeed, many PAHs exhibit such structures and are able to self-assemble into bilayer membranes. For example, isoquinoline or naphthalenecarboxylic derivatives are known to form bilayer structures (Chen et al., 1999).

Depending on the molecular building blocks, self-assembly is driven by weak forces, such as coulombic attractions, $\pi-\pi$ stacking interactions, van der Waals, hydrogen bonding, and hydrophobic forces, or by favorable entropy changes resulting from the release of interfacial water molecules. Small amphiphilic molecules such as longchain fatty acids, amphiphilic quaternary amines, 
and charged PAHs can easily form aggregates in water and consequently assemble into micelles, vesicles, or more complex structures with typical diameters of less than 10 to a few hundred nanometers, depending on conditions $(\mathrm{pH}$, salt concentration, and temperature). Mixed with hydrocarbons or hydrophobic PAHs, the surfactant molecules can encapsulate or integrate more oily substances. In nonpolar media, surfactants can also form inverse micelles around water droplets.

While small lipid molecules of sufficient carbon length may form self-assembling structures, other polymers such as block co-polymers also have a tendency to form stable supramolecular noncovalent assemblies (Discher and Eisenberg, 2002). The building blocks for such polymers (hydrocarbons, carboxylic acids, polyesters, polyamides, oligosaccharides, and polyaromatic compounds) could presumably have been synthesized on early terrestrial planets. As polymeric amphiphilic molecules, these supramolecular noncovalent assemblies can be spontaneously transformed to giant micelles or vesicles with diameters of up to tens of micrometers (Fig. 5).

\section{Possible metabolic chemistry}

Early terrestrial planets have immense free energy reserves available both as sunlight and as chemical redox energy within the crustal minerals and its supply of organics. Chemolitotrophs (organisms that get their energy from oxidation of inorganic compounds) could have emerged with a metabolism based on the nonequilibrium chemical energy in the subsurface minerals. Such simple chemolitotrophic life forms could have harnessed energy from a variety of chemical species such as $\mathrm{S}, \mathrm{H}_{2} \mathrm{~S}, \mathrm{Fe}^{3+}, \mathrm{NH}_{4}{ }^{+}, \mathrm{CO}_{2}$, or $\mathrm{H}_{2}$. Mineral candidates include magnetite $\left(\mathrm{Fe}_{3} \mathrm{O}_{4}\right)$ and maghemite (cubic $\left.\mathrm{Fe}_{2} \mathrm{O}_{3}\right)$, and the iron sulfides pyrite $\left(\mathrm{FeS}_{2}\right)$, greigite $\left(\mathrm{Fe}_{3} \mathrm{~S}_{4}\right)$, and pyrrhotite $\left(\mathrm{Fe}_{7} \mathrm{~S}_{8}\right)$. However, the first life forms may not have been lithoautotrophs, as the primitive Earth most likely had a host of complex organics available. It seems reasonable to assume that the first life forms would have utilized the simplest possible metabolic processes such as fermentation and not more sophisticated energyefficient metabolisms. Later, life may have depleted the initially easily available free energy sources, which would have favored the evolution of more efficient metabolisms.

The large inventories of endogenously generated and extraterrestrially delivered organics

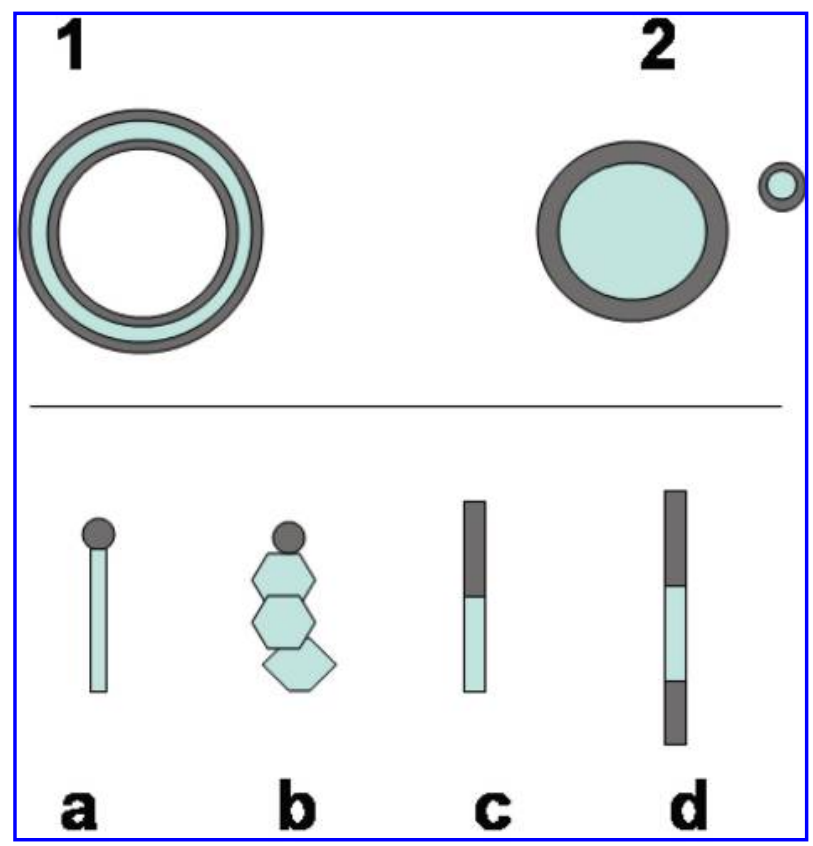

FIG. 5. Possible minimal containers: (1) water-filled bags and (2) hydrophilic bags. In (1), the metabolic and the genetic functionalities reside within the water volume inside the vesicle. In (2), the metabolic and the genetic components are either hydrophobic or amphiphilic and located at the exterior water-lipid interface or within the hydrophobic interior. Note that these container structures can range in size from less than $10 \mathrm{~nm}$ to hundreds of micrometers. (a) A conventional amphiphilic molecule with a polar head group and a non-polar tail. (b) A PAH polymer with a polar head group. (c and d) Block copolymers with one (c) or two (d) hydrophilic sections. The amphiphilic molecule in (a) and certain (b) and (c) polymers can form (bi- or multilayer) vesicles, while all of them can form micelle-like structures (spheres or rods), depending on $\mathrm{pH}$, salt concentration, and other external factors.

could have acted as the earliest metabolic energy sources as well as building blocks. Light energy today is captured by photosynthetically sensitizing systems of plants and prokaryotes. What sensitizer molecules may have been available in the prebiotic environment? Again, PAHs and their derivatives may be good candidates (Deamer, 1992). Many PAHs and their derivatives all absorb light in the near-UV and blue region, and could function to capture light energy, either by donating electrons to produce molecules with higher chemical potential or by generation of ionic gradients. Many aromatic hydrocarbons, fullerenes, and PAHs appear to be capable of capturing or transducing energy (see Table 7). For example, PAHs, such as anthracene and quinones (Chen et al., 1998a, 1999), and other heterocyclic aromatic molecules can be excellent charge trans- 
fer mediators or photosensitizers that might be able to carry out redox or photochemical reactions such as the conversion of lipid precursors to lipid molecules. Fullerenes and their derivatives have been used widely as light-harvesting and electron transfer components in photovoltaic devices (Taylor and Walton, 1993). Since a metabolism functions by transforming resources into building blocks, slightly modifying one type of organic compound into a usable building block may have been the first step in the development of metabolism (Horowitz, 1945), especially if such processes were thermodynamically favorable (Fig. 6).

\section{Possible template chemistry}

A variety of functional amphiphilic containers and energy-capturing and -transduction molecules likely existed on the prebiotic Earth. Templating molecules, however, might be more problematic to synthesize prebiotically. Despite their more complex structure, both simple PAH-based templating polymers and the more sophisticated XNA (e.g., RNA, TNA, GNA, or PNA) polymers could in principle have been the early genetic molecules (Table 7). The self-assembly of XNA polymers from monomers requires polymerization under prebiotic conditions. Most biopolymerization reactions are kinetically unfavorable processes in the absence of specific catalysts such as enzymes, especially in aqueous environments. However, non-aqueous environments, such as a hydrocarbon environment, might shift the polymerization thermodynamics and kinetics sufficiently. Thus a lipid container might be able to mediate XNA polymerization if the XNA is sufficiently lipophilic. This remains to be demonstrated.

A key unresolved question in the origin of life has to do with the early catalytic ability of inheritable molecules. What types of reactions could these early genes have catalyzed? It is more likely that they carried some direct chemical catalysis. In the weakest sense in the context of a protocell, genes are inheritable molecules that control some of the naturally occurring self-assembly and metabolic processes. This concept is at the center of the Los Alamos protocell approach (Rasmussen et al., 2003). In this model, only certain sequences of PNA are assumed to be able to catalyze the reaction of lipid precursors and turn them into surfactant molecules, thus controlling the overall growth of the protocell. For example, templating polymers could serve as the genetic material in the protocell as long as container formation can be controlled by these templating polymers. Thus, chemistry becomes biology when the inheritable molecules control one or more of the container or metabolic functionalities. This means that the templating molecules are catalysts (conceptually similar to ribozymes) that enhance their own fitness within their microenvironment (container + available building blocks) and, thus, their own proliferation.

However, the prebiotic synthesis of XNAs that successively function as the first genetic molecules is questionable because of the structural complexity of these molecules. PAHs might be incorporated more directly into informational polymers, as originally proposed by Platts (e.g., Chapter 17, Hazen, 2005), but we propose a simplified variation on this idea as depicted in Fig. 7, middle. However, an even simpler PAH gene concept can be extracted from what we know about certain polymers: particular types of PAHs interact with each other specifically to form highly ordered liquid crystal phase structures. Hydrogen bonding, $\pi$-stacking, and ionic interactions may mediate the formation of these complex structures (Kato, 2002). A template block polymer with a particular sequence of PAHs might be synthesized (Fig. 7, left). Different neighboring complementary PAH blocks would thus allow a block-specific template replication in functional analogy with the well-known Watson-Crick base pairing (Fig. 7, right). Compared with XNA templating, which is based on precise molecular hybridization, the direct $\mathrm{PAH}$ block polymer templating proposed in Fig. 7, left, is low resolution, yet potentially highly robust, and may be more easily synthesized in prebiotic environments. Experimental demonstration of such templating processes would be extremely interesting.

\section{Integration of the metabolic, genetic, and containment components}

Despite the extensive identified set of possible PAH-based functionalities, it is not at all clear how these components could have self-organized into a minimal living system (see Table 7 and previous subsections). Incompatible component and reaction conditions as well as possible cross-reactions prevent us from simply joining three random structures from the metabolic, genetic, and con- 
FIG. 6. Metabolic reactions 1, 2, and 3 for the generation of functional container-, gene-, and metabolic-building blocks. Resource components 1A, 2A, and $3 \mathrm{~A}$ have to be converted into functional building blocks 1B, 2B, or 3B, respectively, through either the formation or the breakage of a covalent bond. The energy can either be provided as redox or as photo energy. One or more of these metabolic reactions have to be mediated within the protocell container. Minimal life requires an autonomous minimal metabolic system; without available free energy to drive the replication process, however simple it might be, no life is possible. Several cooperative feedbacks are possible; e.g., the hydrophobic microenvironment within the container aggregate could mediate one or more of the metabolic processes. Alternatively, a genetic polymer, such as a ribozyme, could catalyze one or more of the metabolic processes.

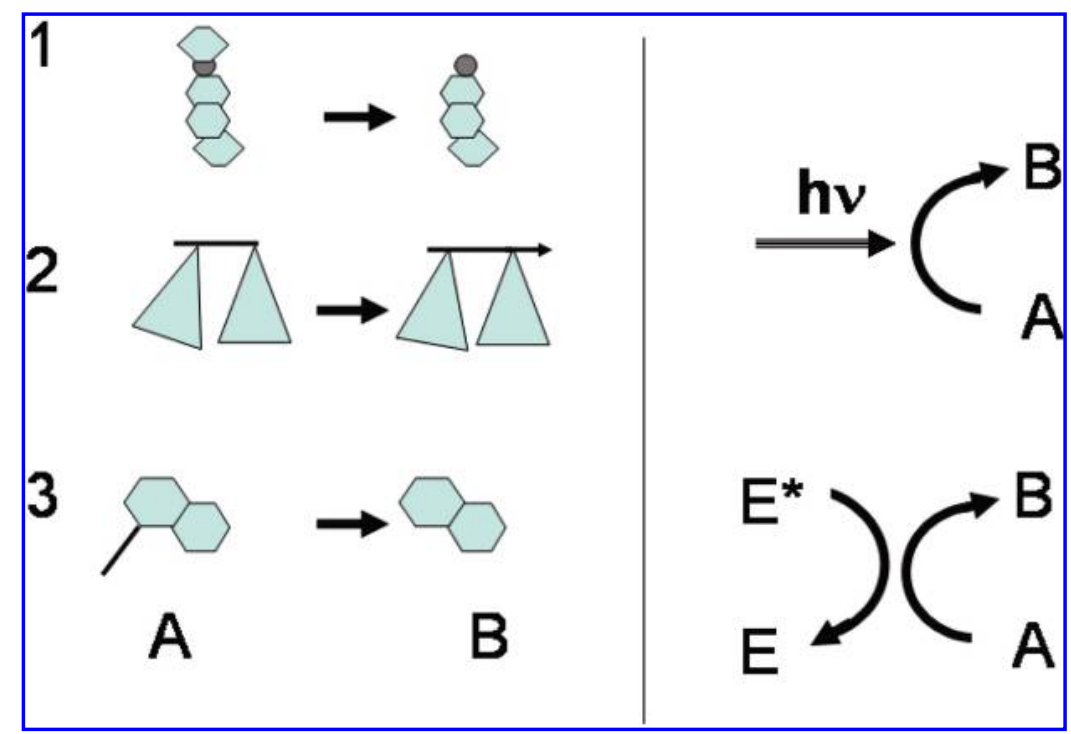

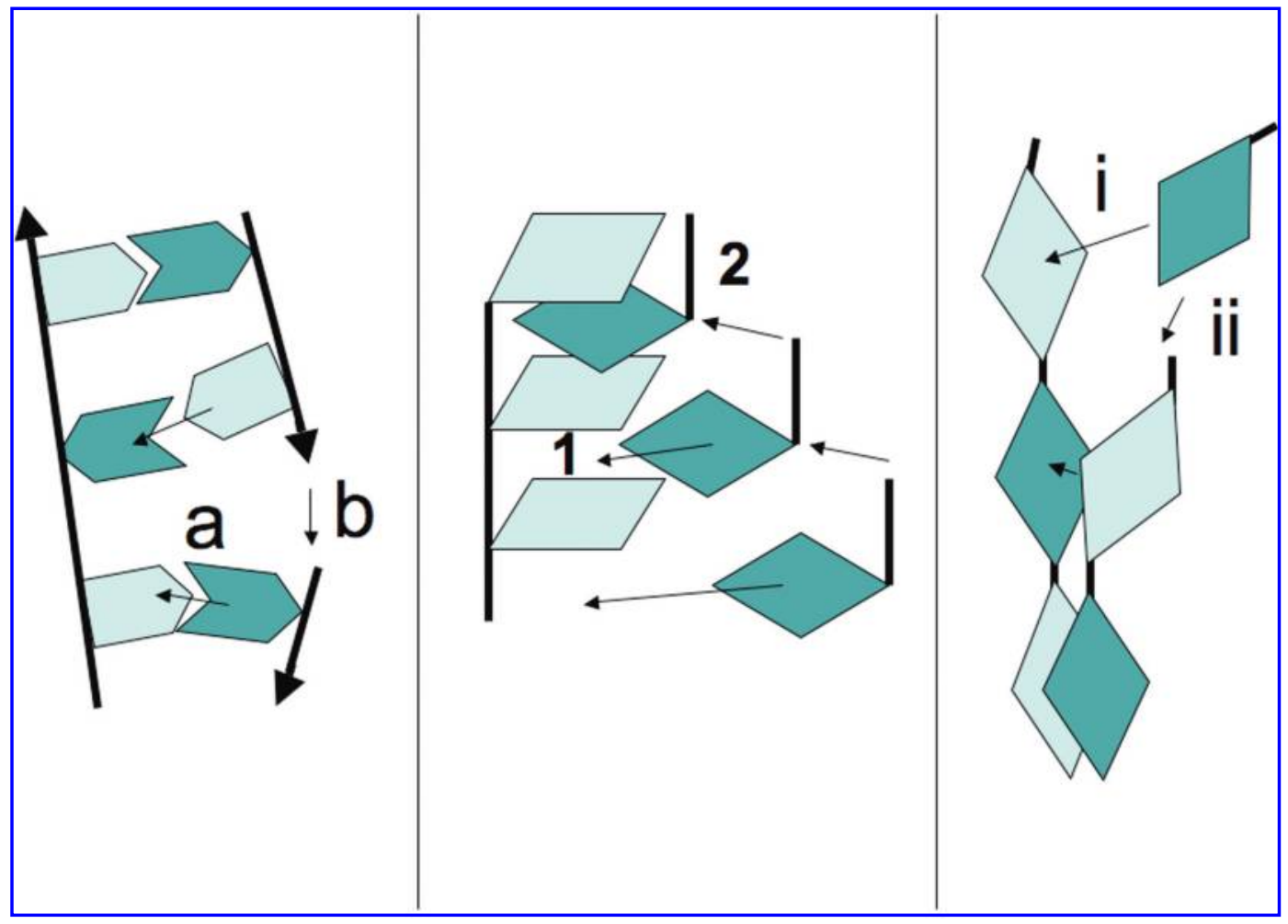

FIG. 7. Some examples of possible early templating polymers. (Left panel) The well-known base recognition process where complementary nucleic bases (XNA, e.g., RNA or PNA) align along an existing template (a) and thus mediate the polymerization (b) of a complementary template. (Middle panel) Example of $\pi$-stacking of particular PAH components [PAH stacking as the basis for an informational polymer was originally proposed by Platts (see Hazen, 2005)] (1), which, in term, could also polymerize (2) to form a complementary template. (Right panel) An even simpler PAH $\pi$-stackingbased templating (i) and polymerization (ii) could occur based on PAH elements that match complementary PAHs already covalently connected into a string. Compared with the modern XNA hybridization, one should expect this proposed linear PAH templating to be less specific, but more readily synthesized in a prebiotic environment. 
tainer sets discussed in the three previous sections. Sufficient conditions for the self-assembly of a (minimal) protocell based on our operational definition are discussed in Fig. 8. A PAH protocell might be able to digest resources and turn them into building blocks that allow the protocell to grow and divide. Furthermore, an imperfect genetic replicator might be able to catalyze part of the digestive, growth, and division processes. A similar organizationally closed protocell structure based on different components is the basis for the Los Alamos protocell (Rasmussen et al., 2003). In fact, all of the proposed protocellular component models are faced with this same integration problem (Morowitz et al., 1988; Luisi et al., 1994; Lee et al., 1996; Szostak et al., 2001; Pohorille and Deamer, 2002; von Kiedrowski et al., 2003). The more theoretically based protocellular approaches traditionally focus on these integration issues, as the theoretical and computational frameworks readily provide a freedom to explore these issues divorced from complex experimental compatibility issues. Many groups have addressed different aspects of these theoretical integration issues both for chem-

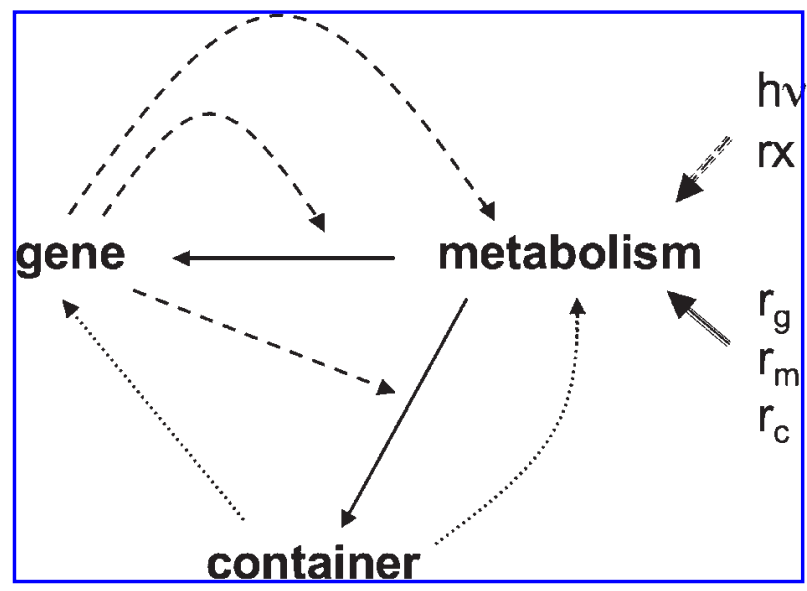

FIG. 8. Sufficient functional interactions that integrate a minimal PAH-based living system (protocell). A lipophilic inheritable replicator (see Possible template chemistry) catalyzes (dashed arrows) the metabolic formation (solid arrows) of both gene- and container-building blocks (see Possible metabolic chemistry). The amphiphilic container molecules self-assemble and increase the container size (see Possible container chemistry). The container ensures a high local concentration and facilitates thermodynamic reaction conditions (dotted arrows) of both the hydrophobic metabolic molecules and the amphiphilic replicator polymers. The necessary free energy can either be redox- or photo-based. Ideally, all three protocellular components should be internally synthesized or directly available from the environment: the resources for the gene monomers, $r_{\mathrm{g}}$, the metabolic complexes, $r_{\mathrm{m}}$, and the container monomers, $r_{\mathrm{c}}$. ical systems and in more abstract settings mostly based on John von Neumann's (1966) and Manfred Eigen's seminal work (Eigen, 1971) (see, e.g., Langton, 1984; Farmer et al., 1986; Kauffman, 1986; Rasmussen, 1988; Bollobas and Rasmussen, 1989; Boerlijst and Hogeweg, 1991; Ganti, 1997; Wächtershäuser, 1997; Sayama, 1998; Segré et al., 2000). In addition to these rational protocell designs, complementary evolutionary design principles are pursued using computer-controlled microfluidics for life support and complementation as well as for combinatorial screening, which is the basis for the PACE project (see http://www.protocells.org/PACE).

Besides the obvious necessary chemical compatibility for component integration, a functional compatibility is necessary as well. Without some coordination in the component formation kinetics, destructive component imbalances may occur. In the Chemoton model of Ganti (2004), the proposed solution is detailed stoichiometric control of the components. Molecular control is imposed such that for each molecule of type $X$ synthesized, a fixed number, $y$, of molecules of type $Y$ are synthesized, while another fixed number, $z$, of molecules of type $Z$ are synthesized, etc. (Munteanu and Sole, 2005). In contrast, the Los Alamos protocell (Rasmussen et al., 2003) defines autocatalytic control between the components, where relative concentrations regulate the component balance. Although catalytic feedback is less restrictive than stoichiometric control, both methods should work, in principle (Rocheleau et al., 2006). However, most published protocell models are not explicit as to how the integrative feedback structures are ensured and, rather, focus more on the experimental issues related to the key protocellular reactions such as template-directed replication and container replication. The insurance of mutual cooperativity between the three main components is the central and likely most difficult experimental protocellular research problem. So far this has not yet been obtained experimentally with any protocell design.

Little is known about the details of the transition from nonliving to living matter on Earth discussed in Fig. 9 or the historical events surrounding it. It is not known whether only one transition occurred, or several transitions converged into a single chemical "solution" (pathway $1 \mathrm{a}$ and $b$ in Fig. 9). It is possible that many transitions (pathways 1, 2, and 3, Fig. 9) could be constructed in the laboratory, some of which 


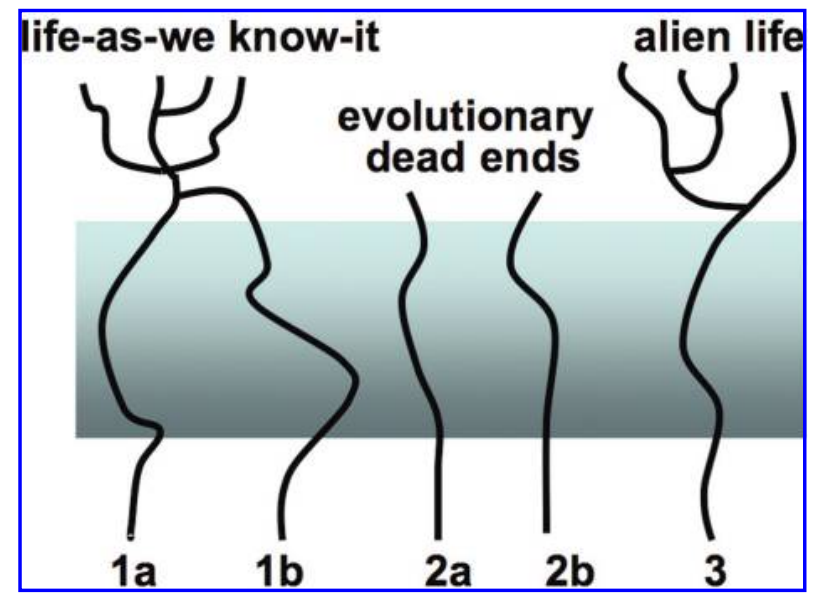

FIG. 9. Many transitions may exist between nonliving and living matter based on our definition of minimal life. To date we know that at least one such transition must have occurred that eventually led to modern life. We propose a PAH-based transition as the most likely candidate. Whether it was a single transition or several transitions that converged into life-as-we-know-it is unknown (pathways 1a and $1 \mathrm{~b}$ ). We hypothesize that many transitions could be constructed in the laboratory that may be compatible with the natural origins of life. Though most of the recently published protocell designs could satisfy the definition of minimal life, they may not be able to evolve any further and thus be unable to converge toward contemporary life. Such evolutionary blind alleys (pathways $2 \mathrm{a}$ and $2 \mathrm{~b}$ ) would still provide us with critical information about what life is, its origin, and possible occurrence elsewhere. Finally, it might be possible to create life forms based on chemistry and an organization that is significantly different from life-as-we-know-it, such that truly alien life could evolve from it (pathway 3). In any event, we propose that studying this transition should be a main focus area of astrobiology.

were alive but could not evolve (pathway 2a and b, Fig. 9). These would then be evolutionary blind alleys, living systems that could only evolve slightly modified simple functionalities but not significant novel functions. Many such evolutionarily limited self-replicating systems have been explored in simulation (Ray, 1991; Lindgren, 1992; Lindgren and Nordahl, 1994; Adami, 1995; Linski et al., 1999). What the minimal system requirements are for open-ended evolution is a key open research question for evolutionary dynamics (Bedau et al., 2000). Today, we have only two documented open-ended evolutionary processes: biological evolution and human technological evolution (Bedau et al., 1998; Skusa and Bedau, 2002).

Different transitions from non-living to living matter should be possible, and alternative routes should be pursued experimentally. It may even be possible to construct living systems based on alternative chemical pathways and organizational principles, which are evolvable. This would be of immense scientific interest, but presently no obvious alternatives exist to carbonbased (bio)chemistry and a cooperation among genetics, metabolism, and container (see, e.g., Bains, 2004; Davies and Lineweaver, 2005). We therefore propose to focus on carbon chemistry. We further propose that the key problem of the origins of life should be tackled experimentally in a step-by-step manner by (1) initially identifying functional container, metabolic, and genetic components followed by (2) a cooperative integration of the three components. At the present time, the astrobiology community has not sufficiently addressed experimentally the critical transition from non-living to living matter.

It may be possible to address several key problems in the origins of life in a rational manner by investigating PAH-based protocell components, which are abundantly distributed throughout our galaxy (see Table 7) and may have the necessary functionalities (stability and flexibility) to serve as the building blocks of life. Why not experimentally explore the most abundant organic molecules on the young Earth as the building blocks for life, aromatic carbonaceous matter?

\section{Proposed experiments}

Many direct tests for container formation are readily possible by studying solvation and selfassembly of a variety of starting materials. For example, the formation of vesicles from different starting materials, including carboxylic acids (Apel et al., 2002) and/or PAH derivatives, should be investigated, and their stability under simulated early Earth conditions such as ocean or subsurface pore space habitats studied. This would provide important constraints for understanding compartmentalization on the young Earth.

Also, simple metabolic processes can directly be tested, e.g., based on PAH-mediated surfactant production. Here we discuss two examples that couple simple prebiotic metabolic processes with the production of container materials. Consider oxidation of hydrocarbons to amphiphilic molecules driven by $\mathrm{PAH}$ derivatives. PAHs have larger electronic delocalization structures (increasing aromaticity) and are stable at the ground state in the dark. However, they can absorb light at 
longer wavelengths (>350 nm, visible light) because of the delocalization structures and, thus, can be easily raised to excited states that have higher oxidation potentials. Consequently, PAHs can photo-oxidize some hydrocarbons to amphiphiles. For example, by using 2-ethyl anthracene, dodecane can be converted into a mixture of dodecanone and dodecanol, both of which are surface-active amphiphiles. Thus, amphiphiles can be generated from hydrocarbons using PAHs and light as an energy source (Klein and Pilpel, 1973; Deamer, 1992).

Another possible metabolic process generates amphiphilic molecules by PAH-sensitized photooxidation. It has been demonstrated that light energy can use low concentrations of oxygen to convert long-chain olefins or PAHs to their polar photo-oxidation products through $\mathrm{PAH}$ sensitization reactions. We have shown that abietic diterpene derivatives can be efficiently oxidized to hydroxy or carbonyl abietic derivatives by perylene photosensitization (Chen et al., 1998b). Significantly, the reaction does not proceed through the conventional photo-oxidation mechanism, which involves singlet oxygen formation, but rather by a novel oxygen interception of a photogenerated exciplex to yield the superoxide. Since many fused PAHs have properties similar to perylene, PAHsensitized oxidation of long-chain alkenes is a plausible pathway for the production of amphiphilic compounds using trace molecular oxygen available on the prebiotic young Earth.

The possible formation of simple PAH-based informational (templating) polymers can also readily be tested. Certain types of PAHs interact specifically with each other to form highly ordered liquid crystal phase structures. For example, molecules such as triphenylene, porphyrin, phthalocyanine, coronene, and other aromatic molecules can form discotic liquid crystals, in which molecules are stacked one-dimensionally and assemble into cylindrical structures because of the $\pi-\pi$ electronic interactions. However, the formation of liquid crystalline phases can also be achieved by hydrogen bonding between amphiphilic molecules bearing complementary functional groups. For example, diaminopyridine and uracil derivatives with long aliphatic tails form liquid crystals (Bricnne et al., 1989). The strong tendency for molecules to form liquid crystal phases renders a cluster of such molecules that can specifically interact with another cluster of similar molecules. Such block-block interactions that form a larger liquid crystal phase will provide an avenue for PAH-based informational templating. Accordingly, a template block polymer with a particular sequence of PAHs with $\pi$ stacking block and hydrogen binding stacking block, respectively, would allow block specific recognitions and hybridization.

To the best of our knowledge, the example described herein is the first suggestion of the preparation of PAH-based templating and hybridization through molecular recognition of block motifs. Further study of the structures of interacting components that lead to stable systems is required. In addition to proper geometry and complementarity, the interacting blocks must also benefit cooperatively, which includes secondary molecular interactions, e.g., similar to the secondary molecular interactions within a DNA duplex that forms and supports the helix structure.

Finally, the structural and chemical changes of aromatic macromolecular matter (as found in carbonaceous meteorites) when exposed to thermal and radiation processing or oxidation need to be investigated in the laboratory. It is important to understand how such material fragments and whether subunits can act as functional building blocks in the origin of life.

The materials used should be similar to compounds found in meteorites, or similar to the possible inventory of early organic compounds. Obviously, a variety of practical issues emerge with this proposal. PAHs are poorly soluble in water, a characteristic that may cause experimental obstacles. However, a variety of organic solvents that could simulate prebiotic hydrocarbons can be used. Also, the hydrophobic nature of PAHs enables them to concentrate in micelles and other aggregates, which could be an advantage, e.g., if the aggregates can be used as reaction containers. The molecular diversity in the extraterrestrially delivered PAHs could also cause problems. However, solutions to these problems may be discovered, as it is already known that many PAHs can function as container elements and energy transduction elements, as well as templating genetic components. Our hypothesis is that it may be possible to assemble a functional protocell using aromatic material.

\section{CONCLUSION}

Carbon-based life as we know it has adapted to a wide range of extreme environments on 
planet Earth. Prebiotic soups resulting from atmospheric synthesis, hydrothermal vents, synthesis from outgassing, and extraterrestrial infall of organic material have been extensively discussed in the last decades (Miller, 1953; Chyba and Sagan, 1992; Russell and Hall, 1997; Gold, 1999; Ehrenfreund et al., 2002). The crucial step in life's origin, the successful assembly of functional components, must be strongly determined by the environment and the ability of organic materials to be concentrated within it (Cleaves and Chalmers, 2004). Besides the presence of organic precursor compounds, water, energy, and catalytic surfaces are among the vital conditions necessary to form more complex structures. While the prebiotic synthesis of many of the constituents of modern biochemical systems has been elucidated, their assembly into a living system remains unsolved. It is possible that chemists will succeed in producing synthetic life using chemicals alien to biochemistry, and it is possible that the earliest terrestrial life was composed of compounds no longer used in modern biochemistry. Precursor molecules that make up our genetic material, such as amino acids, nucleobases, and sugars, have attracted a lot of attention in prebiotic chemistry. However, their stability with respect to the harsh temperature and radiation conditions on the young Earth was very limited. Therefore, it remains an open question as to whether those compounds have been used from the very start to build up life.

In this paper, we have combined knowledge from astrochemistry, prebiotic chemistry, and artificial life in an attempt to construct a model whereby life could have evolved from a different starting material, namely, aromatic hydrocarbons. PAHs and aromatic macromolecules are the dominant organic material in space. Such material may also have been produced terrestrially, and more efficiently than the typical compounds found in modern biochemistry. Aromatic material is extremely stable and quite versatile, and could fulfill different functions by side-group addition and polymerization.

We have elaborated how PAHs might function as container elements, energy transduction elements, and templating genetic components. Our hypothesis is that it is possible to assemble a functional protocell using predominantly aromatic material. We have outlined in Functional Assemblies and Their Integration into a Minimal Life Form how a variety of transitions from nonliving to living matter could be experimentally tested.
Organic chemistry in space seems to follow common pathways throughout the universe. Carbonaceous molecules in the gas or solid state (such as dusty or icy grains) are observed in similar abundances and composition in numerous galaxies, including our own, as discussed in The Formation and Evolution of Organic Material in Space. Aromatic material in the gas phase and in macromolecular form makes up most of the carbon in the interstellar medium, in comets, and in meteorites as well. It is likely that aromatic hydrocarbons are the most abundant organic materials delivered to early planets (Ehrenfreund et al., 2002). Although we cannot exclude the possibility of life based on alien chemistry using neither carbon nor water, the ubiquity of carbon, water, and aromatics across the universe makes it tempting to focus on an origin of life based on aqueous carbon chemistry.

It seems plausible to us that the varied chemistry of diverse macromolecular and aromatic carbon could have played an important role in the origin of life, especially given the stability and likely abundance of such materials on primitive planets. It is even possible that this material was used as the key elements to assemble life, which is why we now propose the "Aromatic World."

\section{ACKNOWLEDGMENTS}

P.E. acknowledges NWO grant 016.023.010. S.R. and L.C. acknowledge Los Alamos LDRD-DR grant on Protocell Assembly and the European Commission grant on Programmable Artificial Cell Evolution (PACE). We thank Nick Cox for graphic support and Mark Sephton for discussion.

\section{ABBREVIATIONS}

FTT, Fischer-Tropsch type; GNA, glycol nucleic acid; HMP, hyper-metal-poor; PAH, polycyclic aromatic hydrocarbon; PNA, peptide nucleic acid; TNA, threose nucleic acid; UV, ultraviolet.

\section{REFERENCES}

Adami, C. (1995) Learning and complexity in genetic auto-adaptive systems. Physica D 80, 154-170.

Allamandola, L.J., Hudgins, D.M., and Sandford, S.A. (1999) Modeling the unidentified infrared emission 
with combinations of polycyclic aromatic hydrocarbons. Astrophys. I. 511, 115-119.

Apel, C.L., Deamer, D.W., and Mautner, M.N. (2002) Selfassembled vesicles of monocarboxylic acids and alcohols: conditions for stability and for the encapsulation of biopolymers. Biochim. Biophys. Acta 1559, 1-9.

Bada, J., Bigham, C., and Miller, S. (1994) Impact melting of frozen oceans on the early Earth: implications for the origin of life. Proc. Natl. Acad. Sci. USA 91, 1248-1250.

Bains, W. (2004) Many chemistries could be used to build living systems. Astrobiology 4, 137-167.

Basiuk, V. and Navarro-Gonzalez, R. (1996) Dust particles in the atmospheres of terrestrial planets and their roles for prebiotic chemistry: an overview. Astrophys. Space Sci. 236, 61-75.

Beaulieu, J.P., Bennett, D.P., Fouque, P., Williams, A., Dominik, M., Jorgensen, U.G., Kubas, D., Cassan, A., Coutures, C., Greenhill, J., Hill, K., Menzies, J., Sackett, P.D., Albrow, M., Brillant, S., Caldwell, J.A., Calitz, J.J., Cook, K.H., Corrales, E., Desort, M., Dieters, S., Dominis, D., Donatowicz, J., Hoffman, M., Kane, S., Marquette, J.B., Martin, R., Meintjes, P., Pollard, K., Sahu, K., Vinter, C., Wambsganss, J., Woller, K., Horne, K., Steele, I., Bramich, D.M., Burgdorf, M., Snodgrass, C., Bode, M., Udalski, A., Szymanski, M.K., Kubiak, M., Wieckowski, T., Pietrzynski, G., Soszynski, I., Szewczyk, O., Wyrzykowski, L., Paczynski, B., Abe, F., Bond, I.A., Britton, T.R., Gilmore, A.C., Hearnshaw, J.B., Itow, Y., Kamiya, K., Kilmartin, P.M., Korpela, A.V., Masuda, K., Matsubara, Y., Motomura, M., Muraki, Y., Nakamura, S., Okada, C., Ohnishi, K., Rattenbury, N.J., Sako, T., Sato, S., Sasaki, M., Sekiguchi, T., Sullivan, D.J., Tristram, P.J., Yock, P.C., and Yoshioka, T. (2006) Discovery of a cool planet of 5.5 Earth masses through gravitational microlensing. Nature 439, 437-440.

Beck, A., Lohrmann, R., and Orgel, L.E. (1967) Phosphorylation with inorganic phosphates at moderate temperatures. Science 157, 952-952.

Becker, L. and Bunch, T.E. (1997) Fullerenes, fulleranes and PAHs in the Allende meteorite. Meteoritics 32, 479-487.

Bedau, M.A., Snyder, E., and Packard, N.H. (1998) A classification of long-term evolutionary dynamics. In Artificial Life VI, edited by C. Adami, R. Belew, H. Kitano, and C. Taylor, MIT Press, Cambridge, MA, pp. 228-237.

Bedau, M.A., McCaskill, J.S., Packard, N.H., Rasmussen, S., Adami, C., Green, D.G., Ikegami, T., Kaneko, K., and Ray, T. (2000) Open problems in artificial life. Artif. Life 6, 363-376.

Beers, T.C. (2005) Where are we now, and where are we going? In ASP Conference Series, Vol. 336: Cosmic Abundances as Records of Stellar Evolution and Nucleosynthesis in Honor of David L. Lambert, edited by T.G. Barnes III and F.N. Bash, Astronomical Society of the Pacific, San Francisco, pp. 283-289.

Beier, M., Reck, F., Wagner, T., Krishnamurthy, R., and Eschenmoser, A. (1999) Chemical etiology of nucleic acid structure: comparing pentopyranosyl- $\left(2^{\prime} \rightarrow 4^{\prime}\right)$ oligonucleotides with RNA. Science 283, 699-703.
Berndt, M.E., Allen, D.E., and Seyfried, W.E. (1996) Reduction of $\mathrm{CO}_{2}$ during serpentinization of olivine at $300^{\circ} \mathrm{C}$ and 500 bar. Geology 24, 351-354.

Bernstein, M.P., Dworkin, J.P., Sandford, S.A., Cooper, G.W., and Allamandola, L.J. (2002) Racemic amino acids from the ultraviolet photolysis of interstellar ice analogues. Nature 416, 401-403.

Bertoldi, F., Cox, P., Neri, R., Carilli, C.L., Walter, F., Omont, A., Beelen, A., Henkel, C., Fan, X., Strauss, M.A., and Menten, K.M. (2003) High-excitation CO in a quasar host galaxy at z $=6.42$. Astron. Astrophys. 409, L47-L50.

Blum, J. (2004) Grain growth and coagulation. In ASP Conference Series 309: The Astrophysics of Dust, edited by A.N. Witt, G.C. Clayton, and B.T. Draine, Astronomical Society of the Pacific, San Francisco, pp. 369-392.

Bockelée-Morvan, D., Crovisier, J., Mumma, M.J., and Weaver, H.A. (2004) The composition of cometary volatiles. In COMETS II, edited by M. Festou, H.U. Keller, and H. Weaver, University of Arizona Press, Tucson, pp. 391-423.

Boerlijst, M. and Hogeweg, P. (1991) Self-structuring and selection: spiral waves as a substrate for prebiotic evolution. In Santa Fe Institute Studies in the Sciences of Complexity, Vol. X: Artificial Life II, edited by C. Langton, C. Taylor, J.D. Farmer, and S. Rasmussen, Addison-Wesley, Redwood, CA, pp. 255-276.

Bollobas, B. and Rasmussen, S. (1989) First cycles in random directed graph processes. Discrete Math. 75, 55-68.

Boss, A.P. (2004) From molecular clouds to circumstellar disks. In COMETS II, edited by M. Festou, H.U. Keller, and H. Weaver, University of Arizona Press, Tucson, pp. $67-80$.

Botta, O. and Bada, J. (2002) Extraterrestrial organic compounds in meteorites. Geophys. Survey 23, 411-467.

Bricnne, M.J., Galard, I., Lehn, J.M., and Stihor, J. (1989) Macroscopic expression of molecular recognition. Supramolecular liquid crystalline phase induced by association of complementary heterocyclic components. I. Chem. Soc. Chem. Commun. 14, 1868-1877.

Brooks, R. (2001) The relationship between matter and life. Nature 409, 409-411.

Butlerov, A. (1861) Formation synthétique d'une substance sucreé. C.R. Acad. Sci. 53, 145-147.

Cairns-Smith, G. (2005) Sketches for a mineral genetic material. Elements 1, 157-161.

Canuto, V., Levine, J., Augustsson, T., and Imhoff, C. (1983) Oxygen and ozone in the early Earth's atmosphere. Precambrian Res. 20, 109-120.

Cataldo, F. (2004) From elemental carbon to complex macromolecular networks in space. In Astrophysics and Space Science Library, Vol. 305: Astrobiology: Future Perspectives, edited by P. Ehrenfreund, W.M. Irvine, T. Owen, L. Becker, J. Blank, J.R. Brucato, L. Colangeli, S. Derenne, A. Dutrey, D. Despois, A. Lazcano, and F. Robert, Kluwer Academic Publishers, Dordrecht, The Netherlands, pp. 97-126.

Cernicharo, J., Heras, A. Tielens, A.G.G.M., Pardo, J.R., Herpin, F., Guèlin, M., and Waters, L.B.F.M. (2001) In- 
frared Space Observatory's discovery of $\mathrm{C}_{4} \mathrm{H}_{2}, \mathrm{C}_{6} \mathrm{H}_{2}$, and benzene in CRL 618. Astrophys. I. 546, 123-126.

Chadha, M. and Choughuley, A. (1984) Synthesis of prebiotic molecules-role of some carbonyl compounds in prebiotic chemistry. Orig. Life 14, 469-476.

Charnley, S., Ehrenfreund, P., and Kuan, Y. (2003) Molecules in space. Physics World Oct, 35-38.

Chen, L., Lucian, L., and Whitten, D.G. (1998a) Cooperative electron transfer fragmentation reactions. Amplification of a photoreaction through a tandem chain fragmentation of acceptor and donor pinacols. L. Am. Chem. Soc. 120, 439-440.

Chen, L., Lucia, L.A., Gaillard, E.R., Whitten, D.G., Icil, H., and Icli, S. (1998b) Photooxidation of a conjugated diene by an exciplex mechanism: amplification via radical chain reactions in the perylene diimide-photosensitized oxidation of $\alpha$-terpinene. L. Phys. Chem. A 102, 9095-9098.

Chen, L., Geiger, C., Perlstein, J., and Whitten, D.G. (1999) Self-assembly of styryl naphthalene amphiphiles in aqueous dispersions and interfacial films: aggregate structure, assembly properties, photochemistry and photophysics. I. Phys. Chem. B 103, 9161-9167.

Cherchneff, I., Barker, J., and Tielens, A. (1992) Polycyclic aromatic hydrocarbon formation in carbon-rich stellar envelopes. Astrophys. J. 401, 269-287.

Chick, K. and Cassen, P. (1997) Thermal processing of interstellar dust grains in the primitive solar environment. Astrophys. J. 477, 398-409.

Choughuley, A., Subbaraman, A., Kazi, Z., and Chadha, M. (1977) A possible prebiotic synthesis of thymine: uracil-formaldehyde-formic acid reaction. BioSystems 9 , 73-80.

Chyba, C. and Sagan, C. (1992) Endogenous production, exogenous delivery and impact-shock synthesis of organic molecules: an inventory for the origins of life. $\underline{\mathrm{Na}}$ ture 355, 125-132.

Chyba, C., Thomas, P., Brookshaw, L., and Sagan, C. (1990) Cometary delivery of organic molecules to the early Earth. Science 249, 366-373.

Cleaves, H. and Chalmers, J. (2004) Extremophiles may be irrelevant to the origin of life. Astrobiology 4, 1-9.

Clemett, S., Maechling, C., Zare, R., Swan, P., and Walker, R. (1993) Identification of complex aromatic molecules in individual interplanetary dust particles. Science 262, $721-725$

Cody, G.D. and Alexander, C.M.O.' (2005) NMR studies of chemical structural variation of insoluble organic matter from different carbonaceous chondrite groups. Geochim. Cosmochim. Acta 69, 1085-1097.

Cody, G., Boctor, N.Z., Filley, T.R., Hazen, R.M., Scott, J.H., Sharma, A., and Yoder, H.S. (2000) Primordial carbonylated iron-sulfur compounds and the synthesis of pyruvate. Science 289, 1337-1340.

Colgate, S., Rasmussen, S., Solem, J., and Lackner, K. (2003) An astrophysical theory for a universal origin of life. Adv. Complex Syst. 6, 487-505.

Cooper, G., Onwo, W.M., and Cronin, J.R. (1992) Alkyl phosphonic acids and sulfonic acids in the Murchison meteorite. Geochim. Cosmochim. Acta 56, 4109-4115.
Corliss, J., Dymond, J., Gordon, L., Edmond, J., von Herzen, R., Ballard, R., Green, K., Williams, D., Bainbridge, A., Crane, K., and van Andel, T. (1979) Submarine thermal springs on the Galapagos Rift. Science 203, 1073-1083.

Corliss, J., Baross, J., and Hoffman, S. (1981) An hypothesis concerning the relationship between submarine hot springs and the origin of life on Earth. Oceanol. Acta 4 (Suppl.), 59-69.

Crovisier, J. (2004) The molecular complexity of comets. In Astrophysics and Space Science Library, Vol. 305: Astrobiology: Future Perspectives, edited by P. Ehrenfreund, W.M. Irvine, T. Owen, L. Becker, J. Blank, J.R. Brucato, L. Colangeli, S. Derenne, A. Dutrey, D. Despois, A. Lazcano, and F. Robert, Kluwer Academic Publishers, Dordrecht, The Netherlands, pp.179-204.

Daigne, F., Olive, K.A., Vangioni-Flam, E., Silk, J., and Audouze, J. (2004) Cosmic star formation, reionization, and constraints on global chemical evolution. Astrophys. L. 617, 693-706.

Dartois, E., Muñoz Caro, G.M., Deboffle, D., Montagnac, G., and D'Hendecourt, L. (2005) Ultraviolet photoproduction of ISM dust. Laboratory characterisation and astrophysical relevance. Astron. Astrophys. 432, 895-908.

Davies, P.C.W. and Lineweaver, C.H. (2005) Finding a second sample of life on earth. Astrobiology 5, 154-163.

Deamer, D. (1985) Boundary structures are formed by organic components of the Murchison carbonaceous chondrite. Nature 317, 792-794.

Deamer, D.W. (1992) Polycyclic aromatic hydrocarbons: primitive pigment systems in the prebiotic environment. Adv. Space. Res. 12, 183-189.

De Graaf, R.M. and Schwartz, A.W. (2005) Thermal synthesis of nucleoside H-phosphonates under mild conditions. Orig. Life Evol. Biosph. 35, 1-10.

Discher, D.E. and Eisenberg, A. (2002) Polymer vesicles. Science 297, 967-973.

Duley, W.W. and Lazarev, S. (2004) Ultraviolet absorption in amorphous carbons: polycyclic aromatic hydrocarbons and the $2175 \AA$ extinction feature. Astrophys. I. 612, 33-35.

Ehrenfreund, P. and Charnley, S.B. (2000) Organic molecules in the ISM, comets and meteorite: a voyage from dark clouds to the early Earth. Annu. Rev. Astron. Astrophys. 38, 427-483.

Ehrenfreund, P. and Fraser, H. (2003) Ice chemistry in space. In NATO ASI Series: Solid State Astrochemistry, edited by V. Pirronello and J. Krelowski, Kluwer Academic Publishers, Dordrecht, The Netherlands, pp. 317-356.

Ehrenfreund, P. and Martin, E.L. (2006) Astronomical constraints for life on terrestrial planets. In COLE Series, Vol. 10: Life as We Know It as: Cellular Origins, Life in Extreme Habitats and Astrobiology, Springer Verlag, New York, in press.

Ehrenfreund, P., Robert, F., d'Hendecourt, L., and Behar, F. (1991) Comparison of interstellar and meteoritic organic matter at $3.4 \mu \mathrm{m}$. Astron. Astrophys. 252, 712-717. Ehrenfreund, P., Irvine, W., Becker, L., Blank, J., Brucato, J., Colangeli, L., Derenne, S., Despois, D., Dutrey, A., 
Fraaije, H., Lazcano, A., Owen, T., and Robert, F. (2002) Astrophysical and astrochemical insights into the origin of life. Rep. Prog. Phys. 65, 1427-1487.

Ehrenfreund, P., Charnley, S., and Wooden, D. (2004) From ISM material to comet particles and molecules. In COMETS II, edited by M. Festou, H.U. Keller, and H. Weaver, University of Arizona Press, Tucson, pp. 115-133.

Eigen, M. (1971) Self-organization of matter and the evolution of macromolecules. Naturwissenshaften 58, 465-523.

Eriksson, M., Christensen, L., Schmidt, J., Haaima, G., Orgel, L., and Nielsen, P. (1998) Sequence dependent $\mathrm{N}$-terminal rearrangement and degradation of peptide nucleic acid (PNA) in aqueous solution. New J. Chem. 22, 1055-1059.

Eschenmoser, A. (2004) The TNA-family of nucleic acid systems: properties and prospects. Orig. Life Evol. Biosph. 34, 277-306.

Farmer, J.D., Kauffman, S., and Packard, N.H. (1986) Autocatalytic replication of polymers. Physica D 22, 50-67.

Ferris, J. (1992) Chemical markers of prebiotic chemistry in hydrothermal systems. Orig. Life Evol. Biosph. 22, 109-134.

Flynn, G.J., Keller, L.P., Feser, M., Wirick, S., and Jacobsen, C. (2003) The origin of organic matter in the solar system: evidence from the interplanetary dust particles. Geochim. Cosmochim. Acta 67, 4791-4806.

Foing, B.H. and Ehrenfreund, P. (1994) Detection of two interstellar absorption bands coincident with spectral features of $\mathrm{C}_{60}{ }^{+}$. Nature 369, 296-298.

Foing, B.H. and Ehrenfreund, P. (1997) New evidences for interstellar $\mathrm{C}_{60}{ }^{+}$. Astron. Astrophys. 317, 59-62.

Frenklach, M. and Feigelson, E.D. (1989) Formation of polycyclic aromatic hydrocarbons in circumstellar envelopes. Astrophys. J. 341, 372-384.

Fuller, W., Sanchez, R., and Orgel, L. (1972) Prebiotic synthesis. VII. Solid-state synthesis of purine nucleosides. I. Mol. Evol. 1, 249-257.

Ganti, T. (1997) Biogenesis itself. J. Theor. Biol. 187, 583-593.

Ganti, T. (2004) Chemoton Theory, Kluwer Academic Publisher, Dordrecht, The Netherlands.

Gardinier, A., Derenne, S., Robert, F., Behar, F., Largeau, C., and Maquet, J. (2000) Solid state CP /MAS ${ }^{13}$ C NMR of the insoluble organic matter of the Orgueil and Murchison meteorites: quantitative study. Earth Planet. Sci. Lett. 184, 9-21.

Gesteland, R., Cech, T., and Atkins, J. (1999) The RNA World, $2^{\text {nd }}$ ed., Cold Spring Harbor Laboratory Press, Cold Spring Harbor, New York.

Goeres, A. and Sedlmayr, E. (1992) The envelopes of R Coronae Borealis stars. I-A physical model of the decline events due to dust formation. Astron. Astrophys. 265, 216-236.

Gold, T. (1999) The Deep Hot Biosphere, Copernicus, New York.

Greenberg, J. (1998) Making a comet nucleus. Astron. Astrophys. 330, 375-380.

Guillois, O., Ledoux, G., and Reynaud, C. (1999) Diamond infrared emission bands in circumstellar media. Astrophys. I. 521, 133-136.
Hayatsu, R., Studier, M., and Anders, E. (1971) Origin of organic matter in the early solar system. IV. Amino acids. Confirmation of catalytic synthesis by mass spectrometry. Geochim. Cosmochim. Acta 35, 939-951.

Hayatsu, R., Studier, M., Matsuoka, S., and Anders, E. (1972) Origin of organic matter in early solar system. VI. Catalytic synthesis of nitriles, nitrogen bases, and porphyrin-like pigments. Geochim. Cosmochim. Acta 36, 555-571.

Hazen, R.M. (2005) Genesis: The Scientific Quest for Life's Origins, Joseph Henry Press, Washington, DC.

Henning, T. and Salama, F. (1998) Carbon in the Universe. Science 282, 2204-2210.

Henning, Th., Jäger, C., and Mutschke, H. (2004) Laboratory studies of carbonaceous dust analogs astrophysics of dust. In ASP Conference Series 309: The Astrophysics of Dust, edited by A.N. Witt, G.C. Clayton, and B.T. Draine, Astronomical Society of the Pacific, San Francisco, pp. 603-628.

Hill, H. and Nuth, J. (2003) The catalytic potential of cosmic dust: implications for prebiotic chemistry in the solar nebula and other protoplanetary systems. Astrobiology 3, 291-304.

Hodgson, G. and Ponnamperuma, C. (1968) Prebiotic porphyrin genesis. Porphyrins from electric discharge in methane, ammonia, and water vapor. Proc. Natl. Acad. Sci. USA 59, 22-28.

Holm, N.G. and Andersson, E.M. (1998) Hydrothermal systems. In Molecular Origins of Life, edited by A. Brack, Cambridge University Press, Cambridge, UK, pp. 86-99.

Holm, N. and Charlou, J. (2001) Initial indications of abiotic formation of hydrocarbons in the Rainbow ultramafic hydrothermal system, Mid-Atlantic Ridge. Earth Planet. Sci. Lett. 191, 1-8.

Horowitz, N. (1945) The evolution of biochemical syntheses. Proc. Natl. Acad. Sci. USA 31, 153-157.

Hotani, H., Lahoz-Beltra, R., Combs, B., Hameroff, S., and Rasmussen, S. (1992) Liposomes, microtubules, and artificial cells. Nanobiology 1, 61-74.

Huber, C. and Wächtershäuser, G. (1998) Peptides by activation of amino acids with $\mathrm{CO}$ on $(\mathrm{Ni}, \mathrm{Fe}) \mathrm{S}$ surfaces and implications for the origin of life. Science 281, 670-672.

Iglesias-Groth, S. (2004) Fullerenes and buckyonions in the interstellar medium. Astrophys. J. 608, 37-40.

Ingar, A., Luke, R., Hayter, B., and Sutherland, J. (2003) Synthesis of cytidine ribonucleotides by stepwise assembly of the heterocycle on a sugar phosphate. ChemBioChem 4, 504-507.

Irvine, W., Crovisier, J., Fegley, B., and Mumma, M.J. (2000) Comets: a link between interstellar and nebular chemistry. In Protostars and Planets IV, edited by V. Mannings, A. Boss, and S. Russell, University of Arizona Press, Tucson, pp. 1159-1200.

Iwamoto, N., Umeda, H., Tominaga, N., Nomoto, K., and Maeda, K. (2005) The first chemical enrichment in the Universe and the formation of hyper metal-poor stars. Science 309, 451-453.

Joyce, G.F. (2002) The antiquity of RNA-based evolution. Nature 418, 214-221. 
Joyce, G.F., Schwartz, A.W., Miller, S.L., and Orgel, L.E. (1987) The case for an ancestral genetic system involving simple analogs of the nucleotides. Proc. Natl. Acad. Sci. USA 84, 4398-4402.

Kaneda, H., Onaka, T., and Sakon, I. (2005) Detection of $\mathrm{PAH}$ emission features from nearby elliptical galaxies with the Spitzer infrared spectrograph. Astrophys. I. 632, 83-86.

Kasting, J. (1993) Earth's early atmosphere. Science 259, 920-926.

Kasting, J. and Catling, D. (2003) Evolution of a habitable planet. Annu. Rev. Astron. Astrophys. 41, 429-463.

Kato, T. (2002) Self-assembly of phase-segregated liquid crystal structures. Science 295, 2414-2418.

Kauffman, S. (1986) Autocatalytic sets of proteins. I. Theor. Biol. 119, 1-24.

Keefe, A. and Miller, S. (1995) Are polyphosphates or phosphate esters prebiotic reagents? I. Mol. Evol. 41, 693-702.

Keefe, A., Miller, S., McDonald, G., and Bada, J.L. (1995) Investigation of the prebiotic synthesis of amino acids and RNA bases from $\mathrm{CO}_{2}$ using $\mathrm{FeS} / \mathrm{H}_{2} \mathrm{~S}$ as a reducing agent. Proc. Natl. Acad. Sci. USA 92, 11904-11906.

Kelley, D.S., Baross, J.A., and Delaney, J.R. (2002) Volcanoes, fluids, and life at mid-ocean ridge spreading centers. Annu. Rev. Earth Planet. Sci. 30, 385-491.

Kelley, D.S., Karson, J.A., Fruh-Green, G.L., Yoerger, D.R., Shank, T.M., Butterfield, D.A., Hayes, J.M., Schrenk, M.O., Olson, E.J., Proskurowski, G., Jakuba, M., Bradley, A., Larson, B., Ludwig, K., Glickson, D., Buckman, K., Bradley, A.S., Brazelton, W.J., Roe, K., Elend, M.J., Delacour, A., Bernasconi, S.M., Lilley, M.D., Baross, J.A., Summons, R.E., and Sylva, S.P. (2005) A serpentinite-hosted ecosystem: the Lost City hydrothermal field. Science 307, 1428-1434.

Klein, A.E. and Pilpel, N. (1973) Oxidation of n-alkanes photosensitized by 1-naphthol. J. Chem. Soc. Faraday I 69,1729-1736.

Kroto, H.W., Heath, J.R., O’Brien, S.C., Curl, R.F., and Smalley, R.E. (1985) C60: buckminsterfullerene. Nature 318, 162-163.

Kuan, Y., Charnley, S.B., Huang, H., Tseng, W., and Kisiel, Z. (2003) Interstellar glycine. Astrophys. I. 593, 848-867.

Kuhn, W. and Atreya, S. (1979) Ammonia photolysis and the greenhouse effect in the primordial atmosphere of the earth. Icarus 37, 207-213.

Kwok, S. (2004) The synthesis of organic and inorganic compounds in evolved stars. Nature 430, 985-991.

Langton, C.G. (1984) Self-reproduction in cellular automata. Physica D 10, 135-144.

Larralde, R., Robertson, M., and Miller, S. (1995) Rates of decomposition of ribose and other sugars: implications for chemical evolution. Proc. Natl. Acad. Sci. USA 92, 8158-8160.

Lee, D., Granja, J., Martinez, J., Severin, K., and Ghadiri, M. (1996) A self-replicating peptide. Nature 382, 525-528.

Levy, M. and Miller, S. (1998) The stability of the RNA bases: implications for the origin of life. Proc. Natl. Acad. Sci. USA 95, 7933-7938.
Li, A. (2005) Can fluffy dust alleviate the subsolar interstellar abundance problem? Astrophys. I. 622, 965-969.

Lindgren, K. (1992) Evolutionary phenomena in simple dynamics. In Santa Fe Institute Studies in the Sciences of Complexity, Vol. X: Artificial Life II, edited by C. Langton, C. Taylor, J.D. Farmer, and S. Rasmussen, Addison-Wesley, Redwood City, CA, pp. 295-312.

Lindgren, K. and Nordahl, M.G. (1994) Evolutionary dynamics of spatial games. Physica D 75, 292-309.

Linski, R.E., Ofria, C., Collier, T.C., and Adami, C. (1999) The minimal systems requirements for open-ended evolution is key open research question for evolutionary dynamics. Nature 400, 661-664.

Lohrmann, R. and Orgel, L. (1971) Urea-inorganic phosphate mixtures as prebiotic phosphorylating agents. Science 171, 490-494.

Luisi, P.L., Giomini, M., Pileni, M., and Robinson, B. (1988) Reverse micelles as hosts for proteins and small molecules. Biochim. Biophys. Acta 947, 209-246.

Luisi, P.L., Walde, P., and Oberholzer, T. (1994) Enzymatic synthesis in self-reproducing vesicles: an approach to the construction of a minimal cell. Ber. Bunsenges. Phys, Chem. 98, 1160-1165.

Luisi, P.L., Veronese, A., and Berclaz, N. (1998) Photoinduced formation of bilayer vesicles. J. Phys. Chem. B 102, 7078-7080.

Maden, B. (1995) No soup for starters? Autotrophy and origins of metabolism. Trends Biochem. Sci. 20, 337-341.

Mannings, V., Boss, A., and Russell, S.S. (2000) Protostars and Planets IV, University of Arizona Press, Tucson.

Marcy, G.W., Butler, R.P., Fischer, D.A., Vogt, S.S., Wright, J.T., and Tinney, C.G. (2005) Observed properties of exoplanets: masses, orbits, and metallicities. Prog. Theor. Phys. Suppl. 158, 24-42.

Markwick, A. and Charnley, S.B. (2004). Chemistry of protoplanetary disks: relation to primitive solar system material. In Astrophysics and Space Science Library, Vol. 305: Astrobiology: Future Perspectives, edited by P. Ehrenfreund, W.M. Irvine, T. Owen, L. Becker, J. Blank, J.R. Brucato, L. Colangeli, S. Derenne, A. Dutrey, D. Despois, A. Lazcano, and F. Robert, Kluwer Academic Publishers, Dordrecht, The Netherlands, pp. 33-66.

Mathis, J.S., Mezger, P.G., and Panagia, N. (1983) Interstellar radiation field and dust temperatures in the diffuse interstellar matter and in giant molecular clouds. Astron. Astrophys. 128, 212-229.

McCollom, T., Ritter, G. and Simoneit, B. (1999) Lipid synthesis under hydrothermal conditions by FischerTropsch-type reactions. Orig. Life Evol. Biosph. 29, 153166.

Mennella, V., Colangeli, L., Bussoletti, E., Palumbo, P., and Rotundi, A. (1998) A new approach to the puzzle of the ultraviolet interstellar extinction bump. Astrophys. J. 507, 177-180.

Miller, S. (1953) A production of amino acids under possible primitive Earth conditions. Science 117, 528-529.

Miller, S. (1957) The mechanism of synthesis of amino acids by electric discharges. Biochim. Biophys. Acta 23, 480-489. 
Miller, S. (1998) The endogenous synthesis of organic compounds. In The Molecular Origins of Life. Assembling Pieces of the Puzzle, edited by A. Brack, Cambridge University Press, Cambridge, UK, pp. 59-85.

Miller, S. and Bada, J. (1988) Submarine hot springs and the origin of life. Nature 334, 609-611.

Miller, S. and Orgel, L. (1974) The Origins of Life on the Earth, Prentice-Hall, Englewood Cliffs, NJ.

Miyakawa, S., Yamanashi, H., Kobayashi, K., Cleaves, H., and Miller, S. (2002a) Prebiotic synthesis from CO atmospheres: implications for the origins of life. Proc. Natl. Acad. Sci. USA 99, 14628-14631.

Miyakawa, S., Cleaves, H., and Miller, S. (2002b) The cold origin of life: A. Implications based on the hydrolytic stabilities of hydrogen cyanide and formamide. Orig. Life Evol. Biosph. 32, 195-208.

Miyakawa, S., Cleaves, H., and Miller, S. (2002c) The cold origin of life: B. Implications based on pyrimidines and purines produced from frozen ammonium cyanide solutions. Orig. Life Evol. Biosph. 32, 209-218.

Mojzsis, S., Arrhenius, G., McKeegan, K., Harrison, T., Nutman, A., and Friend, C. (1996) Evidence for life on Earth before 3,800 million years ago. Nature 384, 55-59.

Mojzsis, S., Harrison, T., and Pidgeon, R. (2001) Oxygenisotope evidence from ancient zircons for liquid water at the Earth's surface 4,300 Myr ago. Nature 409, $178-181$.

Monnard, P.A. and Deamer, D. (2002) Membrane self-assembly processes: steps toward the first cellular life. Anat. Rec. 268, 196-207.

Morowitz, H., Deamer, D., and Heinz, B. (1988) The chemical logic of a minimal protocell. Orig. Life Evol. Biosph. $18,281-287$.

Mosqueira, F., Albarran, G., and Negron-Mendoza, A. (1996) A review of conditions affecting the radiolysis due to $40 \mathrm{~K}$ on nucleic acid bases and their derivatives adsorbed on clay minerals: implications in prebiotic chemistry. Orig. Life Evol. Biosph. 26, 75-94.

Muñoz Caro, G.M., Meierhenrich, U.J., Schutte, W.A., Barbier, B., Arcones Segovia, A., Rosenbauer, H., Thiemann, W.H.-P., Brack, A., and Greenberg, J.M. (2002) Amino acids from ultraviolet irradiation of interstellar ice analogues. Nature 416, 403-406.

Munteanu, A. and Sole, R. (2005) Chaos in chemoton dynamics. In Santa Fe Institute Preprint Series, Santa Fe Institute, Santa Fe, NM, 2005-05-017.

Nelson, K., Levy, M., and Miller, S. (2000) Peptide nucleic acids rather than RNA may have been the first genetic molecule. Proc. Natl. Acad. Sci. USA 97, 3868-3871.

Nielsen, P., Egholm, M., Berg, R., and Buchardt, O. (1991) Sequence-selective recognition of DNA by strand displacement with a thymine-substituted polyamide. $\underline{\text { Sci- }}$ ence 254, 1497-1500.

Nisbet, E. and Sleep, N. (2001) The habitat and nature of early life. Nature 409, 1083-1090.

Nuth, J.A. (1985) Meteoritic evidence that graphite is rare in the interstellar medium. Nature 318, 166-168.

Orgel, L. (1998) The origin of life-a review of facts and speculations. Trends Biochem. Sci. 23, 491-495.
Oró, J. (1960) Synthesis of adenine from ammonium cyanide. Biochem. Biophys. Res. Commun. 2, 407-412.

Oró, J. and Kimball, A. P. (1961) Synthesis of purines under primitive Earth conditions. I. Adenine from hydrogen cyanide. Arch. Biochem. Biophys. 94, 221-227.

Oró, J. and Lazcano, A. (1997) Comets and the origin and evolution of life. In Comets and the Origin and Evolution of Life, edited by P.J. Thomas, C.F. Chyba, and C.P. McKay, Springer, New York, pp. 3-27.

Osterberg, R. and Orgel, L. (1972) Polyphosphate and trimetaphosphate formation under potentially prebiotic conditions. L. Mol. Evol. 1, 241-248.

Pace, N.R. (2001) The universal nature of biochemistry. Proc. Natl. Acad. Sci. USA 98, 808-808.

Pasek, M. and Lauretta, D. (2005) Aqueous corrosion of phosphide minerals from iron meteorites: a highly reactive source of prebiotic phosphorus on the surface of the early Earth. Astrobiology 5, 515-535.

Peeters, Z., Botta, O., Ruiterkamp, R., Charnley, S.B., and Ehrenfreund, P. (2003) The astrobiology of nucleobases. Astrophys. I. 593, 129-132.

Peeters, Z., Botta, O., Charnley, S.B., Kuan, Y., Kisiel, Z., and Ehrenfreund, P. (2005) Formation and photostability of N-heterocycles in space: the effect of nitrogen on the photostability of small aromatic molecules. Astron. Astrophys. 433, 583-590.

Pendleton, Y. and Allamandola, L. (2002) The organic refractory material in the diffuse interstellar medium: mid-infrared spectroscopic constraints. Astrophys. I. Suppl. 138, 75-98.

Pinto, J., Gladstone, G., and Yung, Y. (1980) Photochemical production of formaldehyde in Earth's primitive atmosphere. Science 210, 183-185.

Pohorille, A. and Deamer, D. (2002) Artificial cells: prospects for biotechnology. Trends Biotechnol. 20, 123-170.

Prasad, S.S. and Tarafdar, S.P. (1983) UV radiation field inside dense clouds-its possible existence and chemical implications. Astrophys. J. 267, 603-609.

Rabinowitz, J. and Hampai, A. (1978) Influence of imidazole and hydrocyanic acid derivatives on the "possible prebiotic' polyphosphate-induced peptide synthesis in aqueous solution. Helv. Chim. Acta 61, 1842-1847.

Rasmussen, S. (1988) Toward a quantitative theory of the origin of life. In Artificial Life, edited by C. Langton, Addison-Wesley, Redwood CA, pp. 79-104.

Rasmussen, S., Chen, L., Nilsson, M., and Abe, S. (2003) Bridging nonliving and living matter. Artif. Life 9, 269-316.

Rasmussen, S., Chen, L., Deamer, D., Krakauer, D., Packard, N., Stadler, P., and Bedau, M. (2004) Transitions from non-living to living matter. Science 303, 963-965.

Ray, T.S. (1991) An approach to the synthesis of life. In Artificial Life II, edited by C. Langton, C. Taylor, J.D. Farmer, and S. Rasmussen, Addison-Wesley, Redwood, CA, pp. 371-408.

Reid, C. and Orgel, L. (1967) Synthesis of sugar in potentially prebiotic conditions. Nature 216, 455-455. 
Rietmeijer, F.J.M., Rotundi, A., and Heymann, D. (2004) $\mathrm{C}_{60}$ and giant fullerenes in soot condensed in vapors with variable $\mathrm{C} / \mathrm{H}_{2}$ ratio. Fullerenes Nanotubes Carbon Nanostruct. 12, 659-680.

Robertson, M. and Miller, S. (1995) An efficient prebiotic synthesis of cytosine and uracil. Nature 375, 772-774.

Rocheleau, T., Rasmussen, S., Nielsen, P., Nilsson Jacobi, M., and Ziock, H. (2006) LANL Technical Report: Emergence of Protocellular Growth Laws, LA-UR-05-8916, Los Alamos National Laboratory Technical Report. Proc. R. Soc. Lond. B Biol. Sci. (in press).

Rode, B. (1999) Peptides and the origin of life. Peptides 20, 773-786.

Ruiterkamp, R., Peeters, Z., Moore, M., Hudson, R. and Ehrenfreund, P. (2005a) A quantitative study of proton irradiation and UV photolysis of benzene in interstellar environments. Astron. Astrophys. 440, 391-402.

Ruiterkamp, R., Cox, N.L.J., Spaans, M., Kaper, L., Foing, B.H., Salama, F., and Ehrenfreund, P. (2005b) PAH charge state distribution and DIB carriers: implications from the line of sight toward HD 147889. Astron. Astrophys. 431, 515-529.

Russell, M. and Hall, A.J. (1997) The emergence of life from iron monosulphide bubbles at a submarine hydrothermal redox and $\mathrm{pH}$ front. J. Geol. Soc. Lond. 154, 377-402.

Russell, M.J. and Martin, W. (2004) The rocky roots of the acetyl coenzyme-A pathway. Trends Biochem. Sci. 24, 358-363.

Salama, F., Bakes, E.L.O., Allamandola, L.J., and Tielens, A.G.G.M. (1996) Assessment of the polycyclic aromatic hydrocarbon-diffuse interstellar band proposal. Astron. Astrophys. 458, 621-636.

Sanchez, R. and Orgel, L. (1970) Prebiotic synthesis. V. Synthesis and photoanomerization of pyrimidine nucleosides. L. Mol. Biol. 47, 531-543.

Sanchez, R., Ferris, J., and Orgel, L. (1966a) Conditions for purine synthesis: did prebiotic synthesis occur at low temperatures? Science 153, 72-73.

Sanchez, R., Ferris, J., and Orgel, L. (1966b) Cyanoacetylene in prebiotic synthesis. Science 154, 784-785.

Sanchez, R., Ferris, J., and Orgel, L. (1968) Studies in prebiotic synthesis. IV. The conversion of 4-aminoimidazole-5-carbonitrile derivatives to purines. J. Mol. Evol. $38,121-128$.

Sayama, H. (1998) Introduction of structural dissolution into Langton's self-replicating loops. In Artificial Life VI: Proceedings of the Sixth International Conference on Artificial Life (Complex Adaptive Systems), edited by C. Adami, R.K. Belew, H. Kitano, and C.E. Taylor, MIT Press, Cambridge, MA, pp. 114-122.

Scalo, J. and Biswas, A. (2002) Turbulent compressibility of protogalactic gas. Monthly Notices R. Astron. Soc. 332, 769-776.

Scannapieco, E., Schneider, R., and Ferrara, A. (2003) The detectability of the first stars and their cluster enrichment signatures. Astrophys. I. 589, 35-52.

Schlesinger, G. and Miller, S. (1973) Equilibrium and kinetics of glyconitrile formation in aqueous solution. $[$. Am. Chem. Soc. 95, 3729-3735.
Schlesinger, G. and Miller, S. (1983) Prebiotic synthesis in atmospheres containing $\mathrm{CH}_{4}, \mathrm{CO}$, and $\mathrm{CO}_{2}$. II. Hydrogen cyanide, formaldehyde and ammonia. I. Mol. Evol. 19, 383-390.

Schwartz, A. (1969) Specific phosphorylation of the 2'and $3^{\prime}$-positions in ribonucleosides. I. Chem. Soc. D Chem. Commun. 23, 1393.

Segré, D. and Lancet, D. (2000) Composing life. EMBO Rep. 31, 217-222.

Segré, D., Ben-Eli, D., and Lancet, D. (2000) Composational genomes: prebiotic information transfer in mutually catalytic noncovalent assemblies. Proc. Natl. Acad. Sci. USA 97, 4112-4117.

Segré, D., Ben-Eli, D., Deamer, D.W., and Lancet, D. (2001) The lipid world. Orig. Life Evol. Biosphys. 31, 119-145.

Sephton, M.A. (2002) Organic compounds in carbonaceous meteorites. Nat. Prod. Rep. 19, 292-311.

Sephton, M.A. and Botta, O. (2005) Recognizing life in the Solar System: guidance from meteoritic organic matter. Int. J. Astrobiol. 4, 269-276.

Sephton, M.A., Pillinger, C.T., and Gilmour I. (2000) Aromatic moieties in meteoritic macromolecular materials: analyses by hydrous pyrolysis and ${ }^{13} \mathrm{C}$ of individual compounds. Geochim. Cosmochim. Acta 64, 321-328.

Sephton, M.A., Verchovsky, A.B., and Wright, I.P. (2004) Carbon and nitrogen isotope ratios in meteoritic organic matter: indicators of alteration processes on the parent asteroid. Int. J. Astrobiol. 3, 221-227.

Shapiro, R. (1995) The prebiotic role of adenine: a critical analysis. Orig. Life Evol. Biosph. 25, 83-98.

Shock, E. and Schulte, M. (1998) Organic synthesis during fluid mixing in hydrothermal systems. I. Geophys. Res. Planets 103, 28513-28527.

Shock, E.L., McCollom, T., and Schulte, M.D. (1998) The emergence of metabolism from within hydrothermal systems. In Thermophiles: The Keys to Molecular Evolution and the Origin of Life, edited by J. Wiegel and M.W.W Adams, Taylor and Francis, Washington, pp. 59-76.

Simionescu, B., Mora, R., Leanca, M., and Ananiescu, C. (1980) Genesis of porphyrin-like compounds under simulated abiotic conditions. A possible mechanism of their formation. Rev. Roum. Chim. 25, 1077-1082.

Skusa, A. and Bedau, M.A. (2002) Towards a comparison of evolutionary creativity in biological and cultural evolution. In Artificial Life VIII, edited by R. Standish, M.A. Bedau, and H.A. Abbass, MIT Press, Cambridge, MA, pp. 233-242.

Sleep, N., Meibom, A., Fridriksson, T., Coleman, R., and Bird, D. (2004) $\mathrm{H}_{2}$-rich fluids from serpentinization: geochemical and biotic implications. Proc. Natl. Acad. Sci. USA 101, 12818-12823.

Smith, E. and Morowitz, H. (2004) Universality in intermediary metabolism. Proc. Natl. Acad. Sci. USA 101, 13168-13173.

Snider, M. and Wolfenden, R. (2000) The rate of spontaneous decarboxylation of amino acids. I. Am. Chem. Soc. 122, 11507-11508.

Sollerman, J., Cox, N., Mattila, S., Ehrenfreund, P., Kaper, 
L., Leibundgut, B., and Lundqvist, P. (2005) Diffuse interstellar bands in NGC 1448. Astron. Astrophys. 429, 559-567.

Sowerby, S., Morth, C., and Holm, N. (2001) Effect of temperature on the adsorption of adenine. Astrobiology 1 , 481-487.

Spaans, M. (2004) The synthesis of the elements and the formation of stars. In Astrophysics and Space Science Library, Vol. 305: Astrobiology: Future Perspectives, edited by P. Ehrenfreund, W.M. Irvine, T. Owen, L. Becker, J. Blank, J.R. Brucato, L. Colangeli, S. Derenne, A. Dutrey, D. Despois, A. Lazcano, and F. Robert, Kluwer Academic Publishers, Dordrecht, The Netherlands, pp. 1-16.

Spaans, M. and Silk, J. (2005) The polytropic equation of state of primordial gas clouds. Astrophys. J. 626, 644-648.

Spoon, H.W.W., Moorwood, A.F.M., Pontoppidan, K.M., Cami, J., Kregel, M., Lutz, D., and Tielens, A.G.G.M. (2003) Detection of strongly processed ice in the central starburst of NGC 4945. Astron. Astrophys. 402, 499-507.

Stephan, B. (2002) The chemical basis of membrane bioenergetics. L.Mol. Evol. 54, 595-613.

Stillwell, W. (1977) On the origin of photophosphorylation. I. Theor. Biol. 65, 479-497.

Stribling, R. and Miller, S. (1987) Energy yields for hydrogen cyanide and formaldehyde syntheses: the hydrogen cyanide and amino acid concentrations in the primitive ocean. Orig. Life Evol. Biosph. 17, 261-273.

Szostak, J., Bartel, D., and Luisi, P. (2001) Synthesizing life. Nature 409, 387-390.

Taylor, R. and Walton, D.R.M. (1993) The chemistry of fullerenes. Nature 363, 685-693.

Tian, F., Toon, O., Pavlov, A., and De Sterck, H. (2005) A hydrogen-rich early Earth atmosphere. Science 308, 1014-1017.

Tielens, A.G.G.M., Hony, S., van Kerckhoven, C., and Peeters, E. (1999) Interstellar and circumstellar PAHs. In ESA SP 427: The Universe as Seen by ISO, edited by P. Cox and M.F. Kessler, European Space Agency, Noordwijk, The Netherlands, pp. 579-588.

Tomita, S., Fujii, M., and Hayashi, S. (2004) Defective carbon onions in interstellar space as the origin of the optical extinction bump at 217.5 nanometers. Astrophys.. $609,220-224$.

Tuck, A. (2002) The role of atmospheric aerosols in the origins of life. Surveys Geophys. 23, 379-409.

Umeda, H. and Nomoto, K. (2005) Variations in the abundance pattern of extremely metal-poor stars and nucleosynthesis in population III supernovae. Astrophys. L. 619, 427-445.

van Zuilen, M.A., Lepland, A., and Arrhenius G. (2002) Reassessing the evidence for the earliest traces of life. Nature 418, 627-630.

Verlander, M., Lohrmann, R., and Orgel, L. (1973) Catalysts for the self-polymerization of adenosine cyclic 2',3'-phosphate. L. Mol. Evol. 2, 303-316. von Kiedrowski, G., Eckardt, L., Naumann, K., Pankau, W.M., Reimold, M., and Rein, M. (2003) Toward replicatable, multifunctional, nanoscaffolded machines. A chemical manifesto. Pure Appl. Chem. 75, 609-619.

von Neumann, J. (1966) The Theory of Self Reproducing Automata, edited by A.W. Burks, University of Illinois Press, Urbana.

Wächtershäuser, G. (1988) Before enzymes and templates: theory of surface metabolism. Microbiol. Rev. 52, 452-484.

Wächtershäuser, G. (1997) The origin of life and its methodological challenges. L. Theor. Biol. 187, 483-494.

Weidenschilling, S. and Cuzzi, J. (1993) Formation of planetesimals in the solar nebula. In Protostars and Planets III, edited by E. Levy and J. Lunine, University of Arizona Press, Tucson, pp. 1031-1060.

White, R. (1984) Hydrolytic stability of biomolecules at high temperatures and its implication for life at $250^{\circ} \mathrm{C}$. Nature 310, 430-432.

Wilde, S., Valley, J., Peck, W., and Graham, C. (2001) Evidence from detrital zircons for the existence of continental crust and oceans on the Earth $4.4 \mathrm{Gyr}$ ago. $\underline{\mathrm{Na}}$ ture 409, 175-178.

Wooden, D., Charnley, S., and Ehrenfreund, P. (2004) Composition and evolution of molecular clouds. In COMETS II, edited by M. Festou, H.U. Keller, and H. Weaver, University of Arizona Press, Tucson, pp. 33-66.

Yamagata, Y., Watanabe, H., Saitoh, M., and Namba, T. (1991) Volcanic production of polyphosphates and its relevance to prebiotic evolution. Nature 352, 516-519.

Yan, L., Chary, R., Armus, L., Teplitz, H., Helou, G., Frayer, D., Fadda, D., Surace, J., and Choi, P. (2005) Spitzer detection of polycyclic aromatic hydrocarbon and silicate dust features in the mid-infrared spectra of $\mathrm{z} \sim 2$ ultraluminous infrared galaxies. Astrophys. J. 628, 604-610.

Yuen, G. and Kvenvolden, K. (1973) Monocarboxylic acids in Murray and Murchison carbonaceous meteorites. Nature 246, 301-303.

Zhang, L., Peritz, A., and Meggers, E. (2005) A simple glycol nucleic acid. I. Am. Chem. Soc. 127, 4174-4175.

Zhaxybayea, O. and Gogarten, J.P. (2004) Cladogenesis, coalescence and the evolution of the three domains of life. Trends Genet. 20, 182-187.

Address reprint requests to: Prof. Pascale Ehrenfreund Astrobiology Laboratory

Leiden Institute of Chemistry P.O. Box 9502

2300 RA Leiden, The Netherlands

E-mail: p.ehrenfreund@chem.leidenuniv.nl

http://www.astrobiology.nl 


\section{This article has been cited by:}

1. Elizabeth A. Carter, Matthew A. Pasek, Tim Smith, Terence P. Kee, Peter Hines, Howell G. M. Edwards. 2010. Rapid Raman mapping of a fulgurite. Analytical and Bioanalytical Chemistry 397:7, 2647-2658. [CrossRef]

2. S.E. Maurer , D.W. Deamer , J.M. Boncella , P.-A. Monnard . 2009. Chemical Evolution of Amphiphiles: Glycerol Monoacyl Derivatives Stabilize Plausible Prebiotic MembranesChemical Evolution of Amphiphiles: Glycerol Monoacyl Derivatives Stabilize Plausible Prebiotic Membranes. Astrobiology 9:10, 979-987. [Abstract] [PDF] [PDF Plus]

3. Michael C. Storrie-Lombardi, Jan-Peter Muller, Martin R. Fisk, Claire Cousins, Birgit Sattler , Andrew D. Griffiths , Andrew J. Coates . 2009. Laser-Induced Fluorescence Emission (L.I.F.E.): Searching for Mars Organics with a UV-Enhanced PanCamLaser-Induced Fluorescence Emission (L.I.F.E.): Searching for Mars Organics with a UV-Enhanced PanCam. Astrobiology 9:10, 953-964. [Abstract] [PDF] [PDF Plus]

4. Guido W. Fuchs. 2009. Kreislauf eines kosmischen Überlebenskünstlers. Polyzyklische aromatische Kohlenwasserstoffe im All. Physik in unserer Zeit 40:6, 290-295. [CrossRef]

5. Boncho P. Bonev, Michael J. Mumma, Erika L. Gibb, Michael A. DiSanti, Geronimo L. Villanueva, Karen Magee-Sauer, Richard S. Ellis. 2009. COMET C/2004 Q2 (MACHHOLZ): PARENT VOLATILES, A SEARCH FOR DEUTERATED METHANE, AND CONSTRAINT ON THE CH 4 SPIN TEMPERATURE. The Astrophysical Journal 699:2, 1563-1572. [CrossRef]

6. Sun Kwok. 2009. Delivery of Complex Organic Compounds from Planetary Nebulae to the Solar System. International Journal of Astrobiology 8:03, 161. [CrossRef]

7. V. C. Geers, E. F. van Dishoeck, K. M. Pontoppidan, F. Lahuis, A. Crapsi, C. P. Dullemond, G. A. Blake. 2009. Lack of PAH emission toward low-mass embedded young stellar objects. Astronomy and Astrophysics 495:3, 837-846. [CrossRef]

8. César Menor-Salván, Marta Ruiz-Bermejo, Susana Osuna-Esteban, Guillermo Muñoz-Caro, Sabino Veintemillas-Verdaguer. 2008. Synthesis of Polycyclic Aromatic Hydrocarbons and Acetylene Polymers in Ice: A Prebiotic Scenario. Chemistry \& Biodiversity 5:12, 2729-2739. [CrossRef]

9. Andrew D. Aubrey, John H. Chalmers, Jeffrey L. Bada, Frank J. Grunthaner, Xenia Amashukeli, Peter Willis, Alison M. Skelley, Richard A. Mathies, Richard C. Quinn, Aaron P. Zent, Pascale Ehrenfreund, Ron Amundson, Daniel P. Glavin , Oliver Botta, Laurence Barron, Diana L. Blaney, Benton C. Clark, Max Coleman, Beda A. Hofmann , Jean-Luc Josset , Petra Rettberg, Sally Ride, François Robert, Mark A. Sephton, Albert Yen . 2008. The Urey Instrument: An Advanced In Situ Organic and Oxidant Detector for Mars ExplorationThe Urey Instrument: An Advanced In Situ Organic and Oxidant Detector for Mars Exploration. Astrobiology 8:3, 583-595. [Abstract] [PDF] [PDF Plus]

10. J. L. Bada, P. Ehrenfreund, F. Grunthaner, D. Blaney, M. Coleman, A. Farrington, A. Yen, R. Mathies, R. Amudson, R. Quinn, A. Zent, S. Ride, L. Barron, O. Botta, B. Clark, D. Glavin, B. Hofmann, J. L. Josset, P. Rettberg, F. Robert, M. Sephton. 2008. Urey: Mars Organic and Oxidant Detector. Space Science Reviews 135:1-4, 269-279. [CrossRef]

11. Clifford N. Matthews, Robert D. Minard. 2008. Hydrogen cyanide polymers connect cosmochemistry and biochemistry. Proceedings of the International Astronomical Union 4:S251, 453. [CrossRef]

12. V.N. Kompanichenko. 2008. Three stages of the origin of life process: bifurcation, stabilization and inversion. International Journal of Astrobiology 7:01. . [CrossRef]

13. John Parnell, David Cullen, Mark R. Sims, Stephen Bowden, Charles S. Cockell, Richard Court, Pascale Ehrenfreund, Francois Gaubert, William Grant, Victor Parro , Michel Rohmer, Mark Sephton, Helga Stan-Lotter, Andrew Steele , Jan Toporski , Jorge Vago . 2007. Searching for Life on Mars: Selection of Molecular Targets for ESA's Aurora ExoMars MissionSearching for Life on Mars: Selection of Molecular Targets for ESA's Aurora ExoMars Mission. Astrobiology 7:4, 578-604. [Abstract] [PDF] [PDF Plus]

14. A. G. Donchev, N. G. Galkin, L. B. Pereyaslavets, V. I. Tarasov. 2006. Quantum mechanical polarizable force field (QMPFF3): Refinement and validation of the dispersion interaction for aromatic carbon. The Journal of Chemical Physics 125:24, 244107. [CrossRef]

15. Pascale Ehrenfreund, Mark A. Sephton. 2006. Carbon molecules in space: from astrochemistry to astrobiology. Faraday Discussions 133, 277. [CrossRef] 